\title{
An Artificial-Gravity Space-Settlement Ground-Analogue Design Concept
}

\author{
Gregory A. Dorais ${ }^{1}$ \\ NASA Ames Research Center, Moffett Field, CA, 94035
}

The design concept of a modular and extensible hypergravity facility is presented. Several benefits of this facility are described including that the facility is suitable as a fullscale artificial-gravity space-settlement ground analogue for humans, animals, and plants for indefinite durations. The design is applicable as an analogue for on-orbit settlements as well as those on moons, asteroids, and Mars. The design creates an extremely long-arm centrifuge using a multi-car hypergravity vehicle travelling on one or more concentric circular tracks. This design supports the simultaneous generation of multiple-gravity levels to explore the feasibility and value of and requirements for such space-settlement designs. The design synergizes a variety of existing technologies including centrifuges, tilting trains, roller coasters, and optionally magnetic levitation. The design can be incrementally implemented such that the facility can be operational for a small fraction of the cost and time required for a full implementation. Brief concept of operation examples are also presented.

\section{Nomenclature}

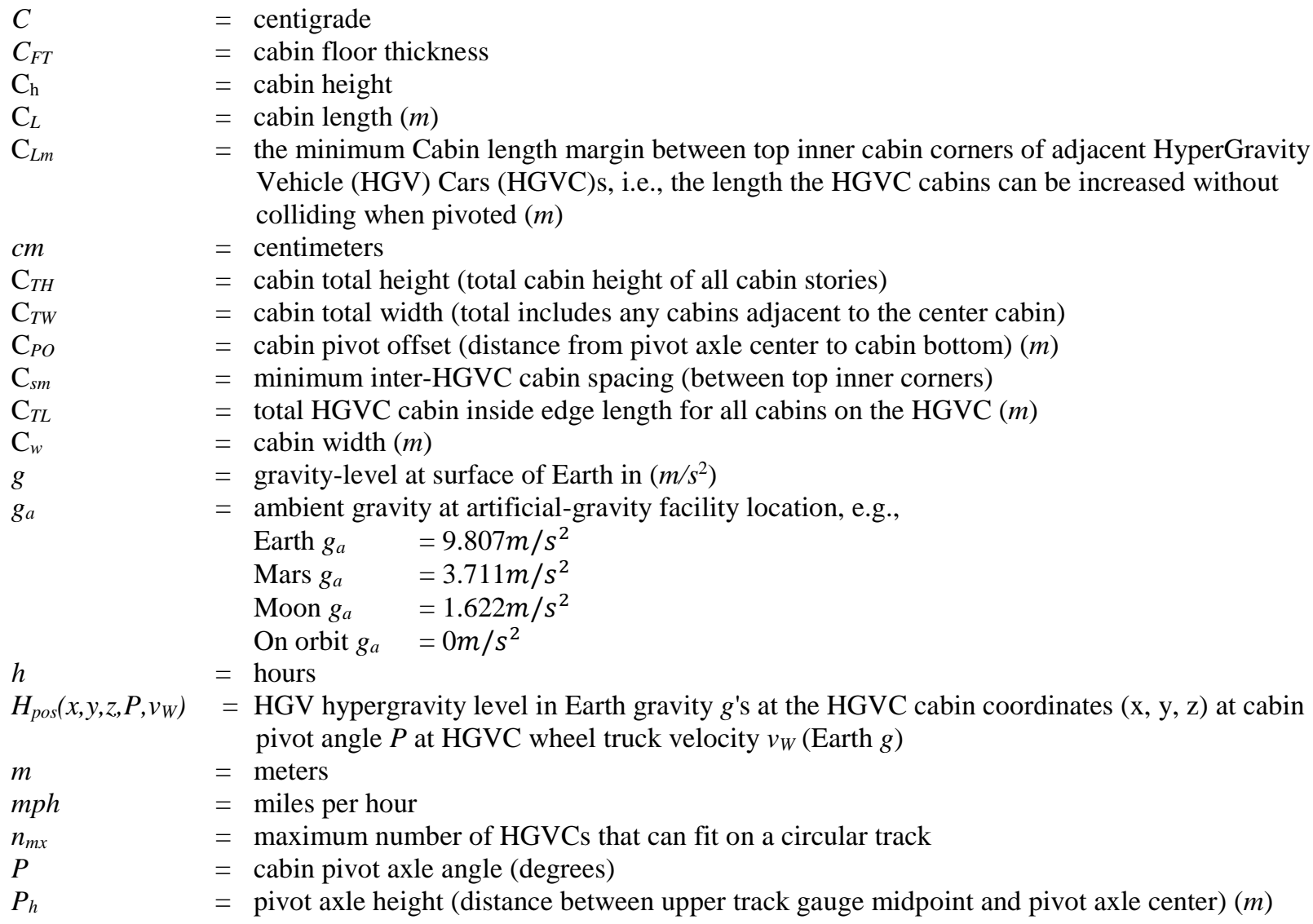

${ }^{1}$ Research Scientist, Exploration Technology Directorate Intelligent Systems Division, MS269-3, AIAA Member 


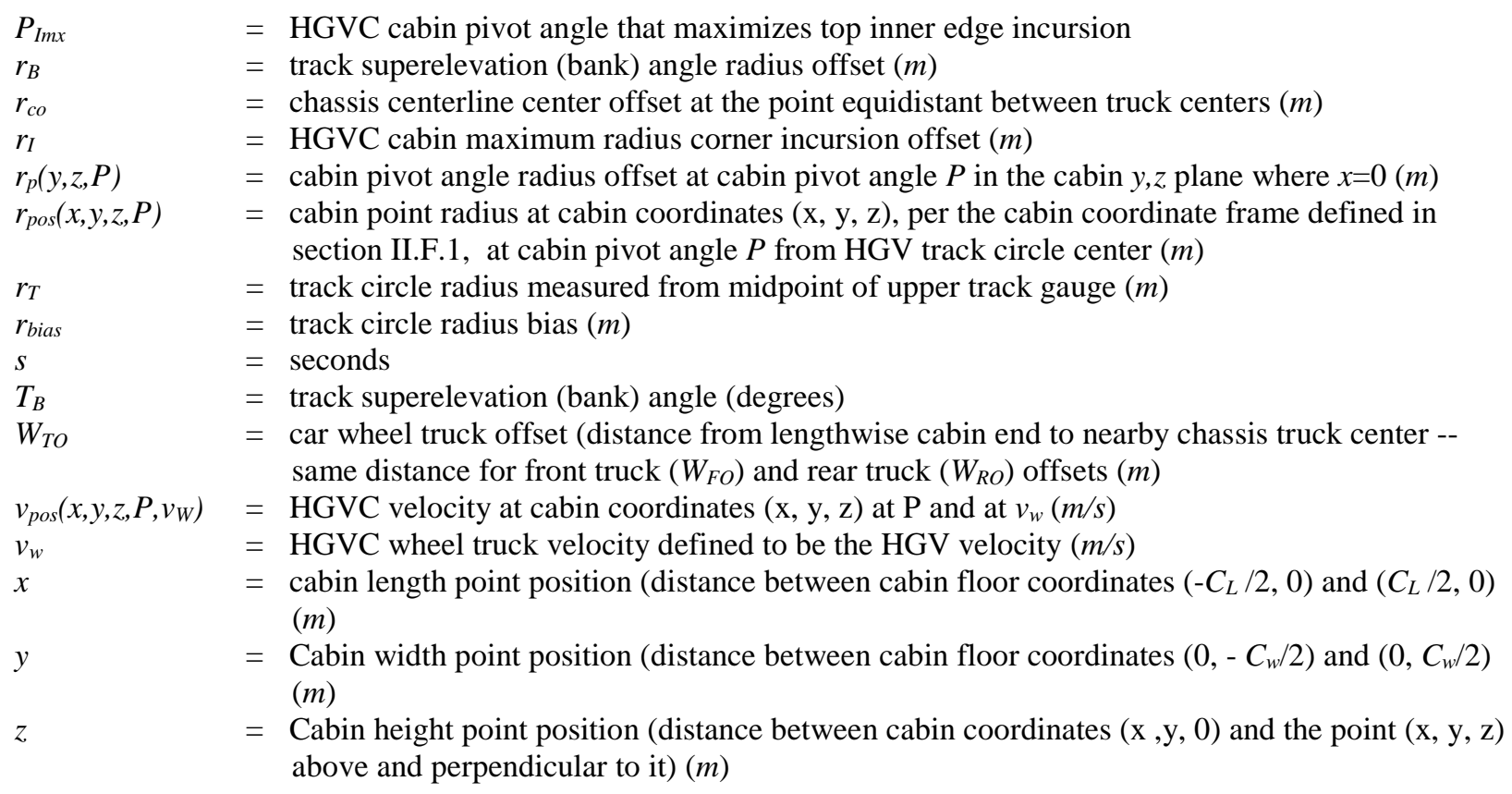

\section{Introduction}

\section{A. Purpose}

This paper defines the design concept for a ground-based, Extended-Stay HyperGravity Facility (ESHGF) as a precursor for space-based artificial-gravity facilities that extend the permanent presence of human, animals, and plants beyond Earth in artificial-gravity settlements. Since the Earth's current human population is stressing the environment and the resources off-Earth are relatively unlimited, by as soon as 2040 more than one thousand people could be living in Earth-orbiting artificial-gravity habitats. Eventually, the majority of humanity may live in artificial-gravity habitats throughout this solar system as well as others, but little is known about the long-term (multi-generational) effects of artificial-gravity habitats on people, animals, and plants.

In order to extend life permanently beyond Earth, it would be useful to create an orbiting space settlement that generates $1 \mathrm{~g}$ as well as other gravity levels to rigorously address the numerous challenges of such an endeavor. Before doing so, developing a ground-based artificial-gravity facility is a reasonable next step. Just as the International Space Station (ISS) is a microgravity research facility, for a small fraction of the cost and risk, a ground-based artificial-gravity facility can begin to address a wide-variety of the artificial-gravity life-science questions and engineering challenges requiring long-term research to enable people, animals, and plants to live offEarth indefinitely.

NASA has been interested in developing such a facility since at least $1969^{1}$ and scientists, such as Dr. Wernher von Braun, were working on the concept decades earlier. In 1952, while von Braun was the Technical Director of the U.S. Army Ordnance Guided Missiles Development Group, the article he authored, "Crossing the Last Frontier," was published, which described the conceptual design of an orbiting space station that would rotate to produce "synthetic" gravity. ${ }^{3}$ The design he envisioned in that article was a 250' diameter wheel, constructed in Low-Earth orbit (LEO) out of modules, and is shown in Fig.1. This space station could produce $1 \mathrm{~g}$ by rotating with a rotational period of 12.3 seconds (4.9 Revolutions Per Minute (RPM)) and produce $1 / 3 g$ by rotating with a rotational period of 22 seconds (2.7 RPM). ${ }^{3}$ Wernher von Braun went on to lead the development of the Saturn $\mathrm{V}$ as the first Center Director for the NASA Marshall Space Flight Center instead.

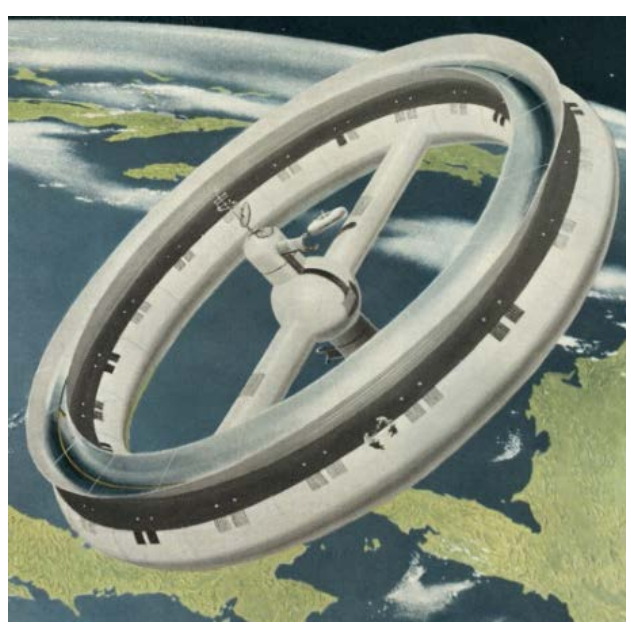

Figure 1. Space Station Design by Wernher von Braun ${ }^{2}$ in 3

American Institute of Aeronautics and Astronautics 
The ground-based extensible and modular ESHGF design concept described in this paper produces an adjustable, hypergravity-level (gravity exceeding $1 \mathrm{~g}$ ) environment in a $150 \mathrm{~m}$ radius rotating facility for people to work and live in along with animals and plants for long-term periods. The design is extensible so that it can be incrementally implemented so that small-scale operations can be realized within a relatively short schedule and relatively low budget. The ESHGF capabilities and capacities can be incrementally increased as demand and budgets permit.

\section{B. Challenges of Extending Life Beyond Earth}

In order to permanently extend life beyond Earth, several challenges must be addressed, a few of which are discussed in this section. For the most part, addressing these challenges has been avoided to lower the cost and complexity of short-term, spaceflight missions.

At the request of the NASA Administrator to make recommendations on the health of astronauts for longduration missions, the National Academies Institute of Medicine committee recommended that NASA focus on giving, "increased priority to understanding, mitigating, and communicating to the public the health risks of longduration missions beyond Earth orbit" and "using more extensively analog environments that already exist and that have yet to be developed;" in 2001. ${ }^{4}$ In 2009, the International Academy of Astronautics Study Group on artificial gravity recommended that, "the most efficient means of developing an effective flight artificial gravity countermeasure is by appropriate and timely use of ground facilities". ${ }^{5}$ The 2012 report by The National Academies National Research Council (NRC) Aeronautics and Space Engineering Board of their review of the NASA Space Technology Roadmap states, "The panel identified Artificial Gravity Evaluation/Implementation as a gamechanging capability that would greatly mitigate many adverse health effects that would otherwise occur during longduration habitation in transit (or Earth orbit)." ${ }^{6}$

Little is known regarding the long-term effects of humans living in artificial-gravity environments. We do know that humans are sensitive to rotations rates, but more research is needed to support artificial-gravity facility design trades. The greater the rotation rate and shorter the centrifuge radius required for humans to be safe and comfortable over long-periods, the lower the mass and cost for the smaller artificial-gravity space facility that is required. The report, "Safe Passage: Astronaut Care for Exploration Missions"4 extensively documents many areas in which knowledge is insufficient regarding the health of astronauts on long-duration missions. Even less is known regarding the effects of various and changing gravity levels on the health of humans living off-Earth indefinitely including child development.

Space settlements may be located at many different locations such as on-orbit, and on the surface or in tunnels in the Moon, Mars, Phobos, Deimos, and asteroids. Each location presents challenges as to what living there might be like and what is necessary to survive under such conditions. By creating artificial-gravity settlements, the negative effects of the natural gravity levels at these locations can be mitigated. By performing research in Earth analogues, the resulting science can inform the design of future spacecraft and space facilities requiring artificial gravity. Doing so can enable the permanent residence of humans off Earth in rotating settlements such that their environments are engineered to be Earth-like or like other off-Earth locations by providing artificial gravity.

With renewed interest in human missions to Mars, ${ }^{7}$ any credible attempt appears to require some type of gravity augmentation due to the extended duration of such missions. To date, only a few people have lived off-Earth for more than a year and the extended time in a microgravity environment caused significant detrimental effects, e.g., muscle loss, cardiovascular deconditioning, neurological anomalies, increased intraocular pressure degrading vision, skin anomalies such as atrophy, and a reduction in bone density. Little is known of the long-term (multi-year and multi-generational) effects for humans, plants, and animals living in a gravity environment of other than $1 \mathrm{~g}$. After a 340 day stay on the ISS ending in 2016, Scott Kelly stated "Exposure to the space environment has permanent effects we simply do not fully understand." in Congressional testimony noting that the effects of prolonged exposure to the micro-gravity, radiation, and atmosphere are of particular concern. ${ }^{8}$ The challenge of perpetually producing "fresh" air in a closed system, in contrast to filtered air scrubbed for $\mathrm{CO}_{2}$, is not trivial, but employing natural means may be helpful. However, such natural means may also depend on the gravity level. An overview of the effects of microgravity on humans and artificial gravity research is presented in "Artificial Gravity". ${ }^{9}$

Creating artificial gravity may solve some problems, but create others if not done properly due to the lack of research on the long-term effects of living in rotating environments, particularly with radii significantly greater than $10 \mathrm{~m}$. Human centrifuges have been tested with radii $<10 \mathrm{~m}$ for short periods, but the short-radius rotational effect is too uncomfortable for humans at more than a few RPM even for short periods, e.g., motion sickness, and the cockpits were too small for long-duration tests.

The "Coriolis effect" is experienced when moving within a rotating reference frame, such as a vehicle or centrifuge, and is associated with vestibular disturbances resulting in motion sickness and disorientation. The physiological effects of Coriolis accelerations with respect to artificial gravity systems are of particular research 
interest. ${ }^{10}$ Negative Coriolis effects are mitigated by constraining head movement and body translation in a centrifuge, but it is undesirable for humans to constrain either of them in a long-term, artificial-gravity facility.

In addition to the Coriolis effect, the gravity gradient of a centrifuge can be a significant issue. A person standing in a centrifuge with an $8 m$ diameter and subject to centripetal acceleration that generates $1 g$ at the feet will only experience approximately $0.5 g$ at the head (excluding Earth gravity). Since the brain contains the vestibular system used to sense gravity and accelerations, such a sharp gravity gradient is disorienting when the person moves, among other things. This gradient decreases as the centrifuge diameter increases and is negligible in a centrifuge with a diameter of $300 m$ that this design concept describes.

Even if it is shown that off-Earth artificial-gravity settlements can provide a safe, long-term environment for their inhabitants, consideration should be given for those travelling between Earth and such settlements for extended periods in microgravity and other off-nominal gravity environments. Space settlements should consider designs with multiple gravity levels to support flight crew training and physical therapy to mitigate the adverse effects of prolonged exposure to low-gravity environments once they arrive and prior to leaving space settlements. By living in a hypergravity environment before and/or after prolonged exposure to low-gravity environments, the negative effects may be mitigated and rehabilitation may be accelerated for humans. ${ }^{11,12}$

Long-term living in a hypergravity environment can increase muscle strength and bone density as well as strengthen organs and improve resistance to loss of consciousness under high-g conditions. Preliminary studies of the effects of prolonged hypergravity on rats, up to $4.7 \mathrm{~g}$ for up to 1 year, resulted in no deleterious effects and discovering significant physiological changes to the rats, such as significant increases in organ and bone mass with little change in overall weight compared to the control rats. ${ }^{13}$ There is also evidence that hypergravity may have therapeutic applications involving cellular development. ${ }^{14}$ Additional research is required to determine the long-term effects of hypergravity on larger mammals as well as the longer-term, multi-generational consequences of hypergravity.

\section{Biomes}

Perhaps the greatest challenge to permanently establishing self-sufficient space settlements off-Earth, will be establishing diverse, viable communities of plant and animals within the settlements. The first space settlements can be expected to rely heavily on support from Earth, but in order to eventually transition to self-sufficiency, consideration of the differences between settlements and ships (or stations) is advised. The most significant differences are the inhabitants themselves, the duration of their stay, and their support. On a ship, trips can last hours to months, the crew members are selected for their expertise, and they are usually supported by preserved foods. In a settlement, the inhabitants can be expected to vary widely from those in ships with respect to expertise, age, health, and relationships, e.g., families; their stays can be for life, and the efficient production of food and recycling of resources becomes imperative.

In "Bio-Ecology"15, Clements and Shelford first defined the term "biome" essentially as a biotic community of plants and animals populating a region. They stated, "From the beginnings of life, organisms have lived together in some kind of grouping . ... We know now that there are no habitats in which both plant and animal organisms are able to live, in which both do not occur and influence each other." ${ }^{56}$ In the long-term, it takes more than a community for humans to survive, it takes a biome.

A minimal biome required to sustain human life over the long-term, let alone a healthy quality of life for humans, is not well understood in a $1 g$ environment, and much less so in other gravity environments. People can live for months by relying on preserved foods from Earth, which may be fine for exploration and mining ships. However for permanently supporting life in settlements beyond Earth, the inhabitants could achieve a measure of self-sufficiency and increased quality of life by naturally recycling air, water, and organic waste; harvesting their own fresh food; as well as by living in natural environments among plants and animals;

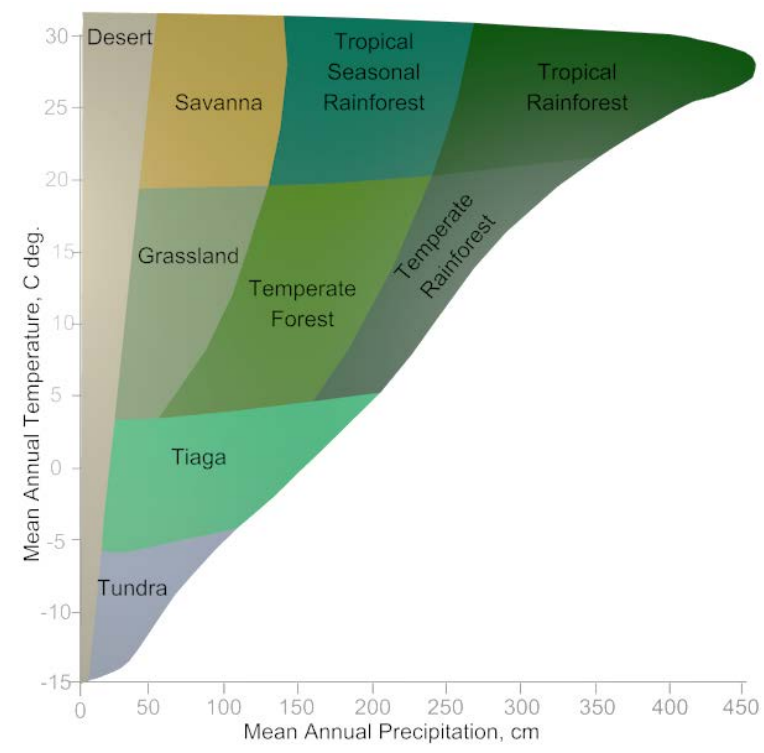

Figure 2. Whittaker Biome Diagram derivation ${ }^{15}$ 
including companion animals, all of which are challenging tasks.

In Communities and Ecosystems, ${ }^{16}$ Whittaker characterized several biomes with respect to the mean temperature and mean precipitation. A simplified version of Whittaker's diagram is shown in Fig. 2. Throughout the world, the same types of species tend to group together where the environments are similar.

Although it is possible for many species to survive individually in environments with mean temperature and precipitation levels they are not well adapted for, they cannot compete with other species and are naturally eliminated reinforcing biome-specific species. Most significantly, very little is known regarding the longterm (multi-generational) effects of gravity levels either above or below $1 g$ on all known life and their biomes as depicted for terrestrial biomes in Fig. 3.

In addition, life seems to benefit from variations in temperature and precipitation daily and annually; and since humans and animals benefit from alternate periods

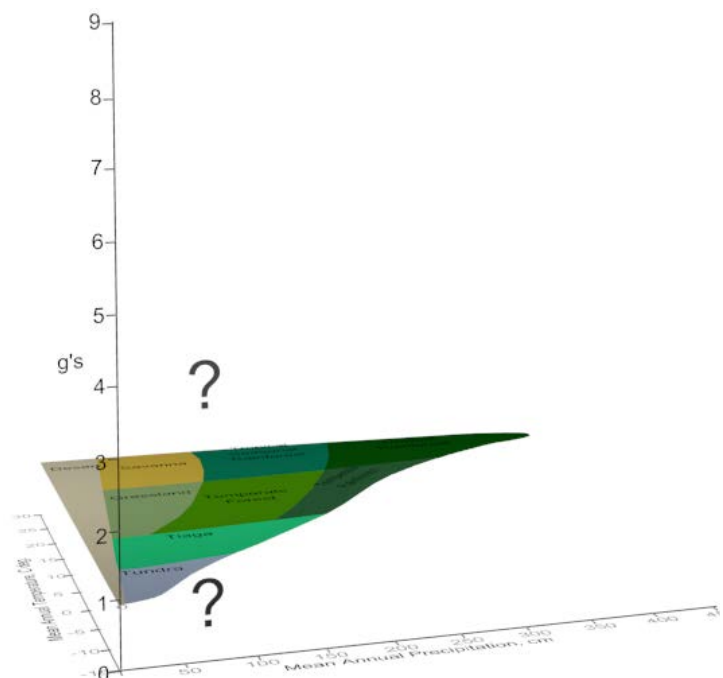

Figure 3. 3D Whittaker Biome Diagram with gravity z-axis added of exercise and rest, life may also may benefit from varying levels of gravity given the opportunity. Since most life appears to be able to survive at gravity levels above and below $1 \mathrm{~g}$ for at least months, albeit with the significant short-term effects, along with the general adaptability of life, the long-term effects can reasonably be expected to be even more significant.

\section{Benefits}

The ESHGF is expected to yield several benefits that can be used to address the above challenges:

- Validate artificial-gravity facility designs on Earth as precursors for their development off-Earth.

- Use as an analogue for off-Earth settlements.

- Characterize the effects and risks of long-term and periodic exposure to various hypergravity levels on humans, animals, and plants in a closed, engineered environment.

- Characterize the long-term effects of living in rotating environments with radii significantly greater than $10 \mathrm{~m}$.

- Characterize the long-term effects artificial gravity of on a wide variety of biomes ranging from desert to oceanic with particular regard to their uses in enabling self-sufficient settlements as well as preserving endangered species in their native habitats.

- Provide flight-crew training and physical therapy to mitigate the effects of prolonged exposure to the microgravity environments on the ISS.

- Provide physical therapy to improve human and animal health and capabilities. Once validated for prolonged use by humans, such facilities could be used for individuals who would benefit from extraordinary strength, e.g., athletes, and select military personnel, or simply to improve their fitness and accelerate weight loss without requiring any special activities, such as while sleeping. It also could be used for physical therapy for patients unable to exercise, e.g., subject to extended bed rest, and for elderly individuals to mitigate muscle and bone loss.

- Validate the human use of a wide-variety of systems that were engineered and tested for use on Earth at $1 g$, e.g., shower, toilet, blender, washing machine, refrigerator, since their performance can vary significantly at different gravity levels and rotation rates. Enabling the development of $1 \mathrm{~g}$ space settlements significantly reduces or entirely eliminates the cost and risk of reengineering the wide variety of objects and systems they require.

\section{Background}

The ESHGF is essentially the merger of two technologies: centrifuges and trains, in which an extremely longarm centrifuge is created using vehicles similar to tilting trains. 


\section{Centrifuges}

Currently, an $18 m$ radius centrifuge operates at the Yuri Gagarin Research \& Test Cosmonaut Training Center that is capable of generating up to $8 g$, has three interchangeable cabins mounted at the end of the arm, and is used for space crew training. ${ }^{17}$ As shown in Fig. 4, a 29' radius $20 \mathrm{~g}$ centrifuge (human-rated for $12.5 \mathrm{~g}$ ) operates at the NASA Ames Research Center and has exposed human subjects to hypergravity for durations up to 22-hours. ${ }^{18}$ Both centrifuges have relatively small cabin sizes and the occupants generally are intended to remain stationary within the cabins during operation.

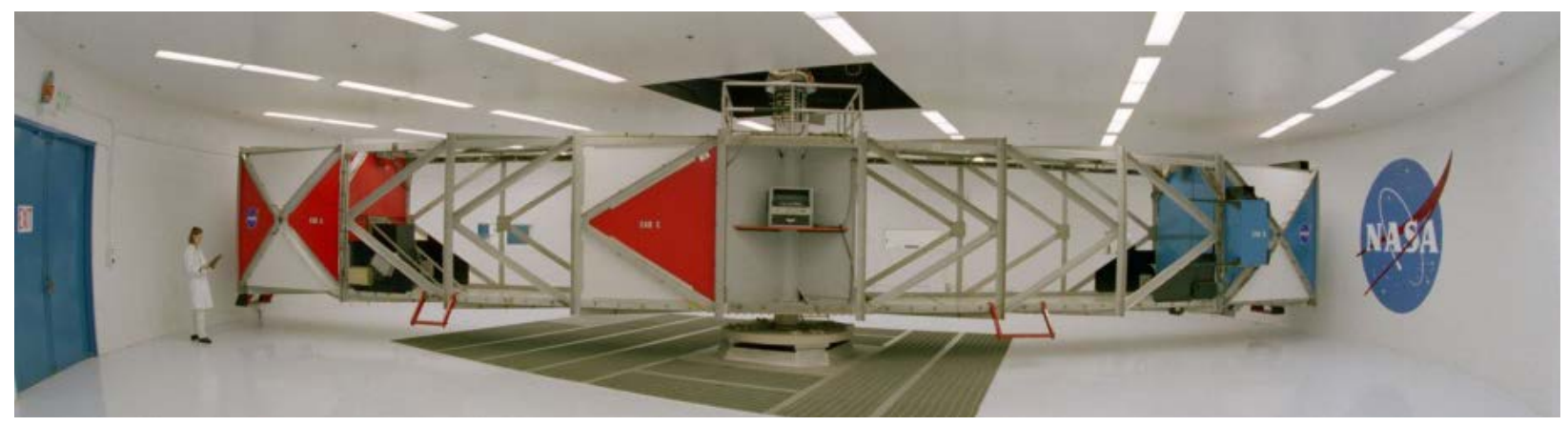

Figure 4. NASA Ames Research Center $20 g$ Centrifuge $^{18}$

Human centrifuges have been used to generate hypergravity for centuries. Erasmus Darwin recounted experiments in which the human subject was placed on a rotating corn-mill stone, which was accelerated to an RPM that mechanically induced "sleep". ${ }^{19}$ Centrifuges were used in psychiatric wards to treat patients in the early 1800s. ${ }^{20}$

In 1895, Konstantin Tsiolkovsky, a pioneer of astronautics, wrote "Dreams of Earth and Sky," in which he imagined generating artificial gravity in space by means of trains running in circles. ${ }^{21,22}$ Later in "Investigation of World Spaces by Reactive Vehicles (1911-1912)," he noted that the further a human subject is from the axis of a centrifuge, the better. Tsiolkovsky also experimented with centrifuges on animals and proposed that a train on a tilted, circular track could be used to generate artificial gravity over extended periods for research. ${ }^{23}$ However, there is no record that such a train was ever designed or built.

Starting in the 1950s, humans were tested in a centrifuge with a 50' arm, capable of generating close to 50g's, at the Naval Air Development Center in Johnsville, PA. In 1958, this facility was used to test X-15 pilots and later was used for astronaut testing for the NASA Mercury, Gemini, and Apollo space programs. ${ }^{24}$ In 1959, the Army Ballistic Missile Agency proposed a human “Space Flight Simulator” that consisted of a 500'-radius superelevated circular track, a 4-person 24' jet-powered sled, a transition track, and a 3622' vertical track configured such that the sled would ascend and launch into a sub-orbital trajectory after reaching a sufficient velocity by traveling on the circular track. ${ }^{25}$

In the 1975 NASA study, "Space Settlements: A Design Study,"27 an orbiting space settlement capable of supporting a population of ten thousand is described in detail. The orbiting space settlement envisioned in this study is shown in Fig. 5. In this design, the $1790 \mathrm{~m}$ diameter space station wheel rotates at 1 RPM inside a non-rotating ring shield to produce $1 \mathrm{~g}$ at the wheel rim bottom. The wheel rim modules can be viewed as train cars running on a circular track in which the track is the ring shield.

Thomas W. Hall's doctoral dissertation, "The Architecture of Artificial-Gravity Environments for Long-Duration Space Habitation," describes several design considerations with regard to

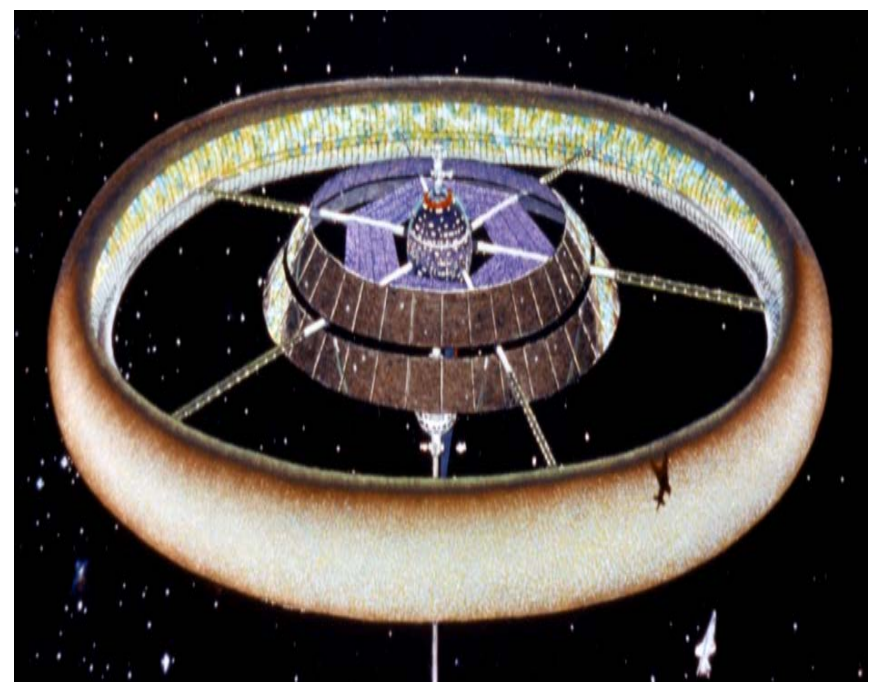

Figure 5. Orbital Space Settlement Design ${ }^{26}$ 
designing facilities for long-term occupation that rely on spin-induced centripetal acceleration to generate artificial gravity. ${ }^{28}$

A long-duration, large-radius "Human Hypergravity Habitat" was proposed..$^{29,30,31}$ The concept is essentially a train on a circular track designed to generate a specific, long-duration hypergravity level for humans. The European Space Agency Large Radius Human Centrifuge Topical Team recommended a track diameter of $150 \mathrm{~m}$ with a maximum design load of $2 g$ 's. Initial tests for long-term hypergravity exposure should be in the range of $1.4-1.5 \mathrm{~g}$ 's and then incrementally increased. ${ }^{30}$

\section{Trains}

An ESHGF can be implemented using readily available wheeled-train technologies. Trains have been used for over two centuries for reliable transport and are operational around the world. They remain a dominant, low-cost means of transportation.

Richard Trevithick is credited with building the first steam-powered train. It was based on a portable steam engine patented by Trevithick and Andrew Vivian in 1802,32 and ran 9 miles at nearly 5 miles per hour carrying 10 tons of iron, 5 wagons, and 70 men in South Wales on 2/21/1804; heralding a new age of transportation. ${ }^{33,34}$

\section{a. $\quad$ Tilting Trains}

Trains with tilting cabins to compensate for track cant as well as centripetal acceleration on curves began being developed in the mid-1930s. ${ }^{35,36}$ These first tilting cabins were mounted on their rail car chassis with pivot points above their cabin centers of gravity and passively pivoted due to gravity and inertia. During the same period, actively-controlled train cabins were designed to tilt hydraulically. ${ }^{37,38}$ Although actively controlled tilting train cabins were not put into operation until decades later, cabin tilting technology is now considered mature. As of 2007, over 5,000 tilting-cabin train cars have been produced worldwide by several suppliers. ${ }^{39,40}$

\section{b. Roller Coasters}

Although tilting trains can compensate for some centripetal acceleration and track cant, a different technology was developed for trains designed to operate with high levels of centripetal acceleration and steep track banks, which has been primarily used for roller coasters. The first modern roller coaster using tubular steel rails was developed by Karl Bacon and Edgar Morgan at Arrow Development for Walt Disney Productions. Their patent for this invention states the purpose was to, "carry passengers at high speeds with absolute safety over a path that includes very sharp and highly banked curves, such as are found in a bobsled course." 41 This technology was first instantiated in the Matterhorn Bobsled roller coaster at Disneyland, which began operating in 1959. Karl Bacon also patented the first "corkscrew" roller coaster using tubular steel rails, which began operating at the Knott's Berry Farm amusement park in $1975 .{ }^{42,43}$ Variations of their looping tubular steel rail roller coasters continue to be used worldwide with the fastest commercial roller coaster in 2015 being the Formula Rosso at Ferrari World Abu Dhabi, United Arab Emirates, which reaches $240 \mathrm{~km} / \mathrm{h}(149 \mathrm{mph})$ on a $2.07 \mathrm{~km}$ track. ${ }^{44}$

\section{c. Locomotion}

Currently, nearly all trains use rotating electric motors in the cars or in the tracks to provide the torque required to rotate the wheels to move the trains. The electric motors in the cars are called electric traction motors and usually get their power from either diesel generators also located in the cars or from electric lines that run along the track or overhead. On some trains, only cars dedicated for locomotion (locomotives) have electric traction motors. An example of an electric locomotive currently being used is the Amtrak Cities Sprinter ACS-64. ACS-64s were adapted for service in the United States for speeds up to $201 \mathrm{~km} / \mathrm{h}(125 \mathrm{mph})$ and are being used on the Amtrak North East Corridor between Boston and Washington D.C. ${ }^{45}$ However, on some trains each car has one or more electric traction motors to distribute the force required to move the trains providing redundancy and scalability. For some trains, such as many roller coasters, electric motors are attached on or nearby the track and used to propel the train, at least to a just beyond a high point after which gravity provides the required acceleration.

Linear motors are becoming more popular for use on trains. Unlike traditional electric motors in which the rotor rotates inside a stator to produce a torque used to turn wheels to produce a linear force, a linear motor in effect flattens the rotor and stator and directly produces a linear force without any moving parts. Linear motors are particularly attractive for magnetically levitated trains in which part of the motor is attached to the bottom and/or sides of the train and the other half is attached to the track. ${ }^{46}$ 


\section{d. Magnetic Levitation}

Ideally, an ESHGF would use superconductor magnetic levitation, which would provide a smooth, quiet, low friction, low-maintenance, and low energy-cost motion. The first patent for a linear induction motor for driving trains was granted to Alfred Zehden in $1905^{47}$ followed by a patent granted to Emile Bachelet for a magnetic levitation transportation apparatus. ${ }^{48}$ In 1969, a patent for a Maglev train that uses superconducting magnets was granted to James Powell and Gordon Danby. ${ }^{49}$ Subsequently, with an operational speed of $430 \mathrm{~km} / \mathrm{h}(267 \mathrm{mph})$ (without superconducting magnets), 464-seat Maglev trains in Shanghai began operations in 2003. ${ }^{50,51}$ The Central Japan Railway is developing a superconducting maglev train. ${ }^{52}$ On April 21, 2015 on the Yamanashi Maglev Line, an $18.4 \mathrm{~km}$ test track, the L0 Series A07 superconducting maglev train ran at $603 \mathrm{~km} / \mathrm{h}(375 \mathrm{mph}) .{ }^{53}$ Although the high-speeds of superconducting maglev trains are not required by a HyperGravity Vehicle (HGV), the other benefits mentioned above are significant.

Another magnetic levitation alternative for consideration is based on the Inductrack developed at the Lawrence Livermore National Laboratory. Unlike the above technologies, which use electromagnets for levitation, the Inductrack, patented by Richard Post, ${ }^{54}$ uses Halbach arrays of permanent magnets for levitation. ${ }^{55}$ This research was sponsored in part by NASA to demonstrate the feasibility of using the Inductrack to accelerate a launch vehicle model at $10 \mathrm{~g}$ to Mach 0.4 .

Taking the next technological step in high-speed magnetic levitation, the "StarTram" concept has been proposed as a low-cost means to put cargo in orbit using superconducting magnetic levitation and linear motors. ${ }^{56,57}$ For offEarth space settlements requiring thousands of modules, the development of this type of infrastructure is worth further consideration. Once demonstrated on Earth, it could also be used on the Moon, which requires 1/6th the escape velocity and doesn't have an atmosphere that would significantly decelerate a launched payload, with or without passengers. This approach will be of extraordinary value in any significant effort to launch people and cargo from the Moon. Since no propellant is required, propellant won't have to be brought all the way from the Earth and safely landed on the Moon (or mined and processed from its polar craters).

\section{Design Concept}

Leveraging the above centrifuge and train technologies, the following design concept describes a modular ESHGF that can be initially implemented to support a few people, but can be incrementally extended as needed to reach a fullscale space settlement capable of supporting biomes with and without people. This section begins with a brief system overview followed by a description of each subsystem. Hypergravity implications are discussed in section II.F, including its effect on system sizing. This is followed by a description of ESHGF stability considerations, modularity, system capacity, and extensibility.

\section{A. System Overview}

The ESHGF system consists of the HyperGravity Vehicle, Vehicle Track, Transfer Vehicle (optional), and Depot subsystems as shown in Fig. 6, which interact as depicted in Fig. 7.

Each of these subsystems are discussed further in the following subsections.

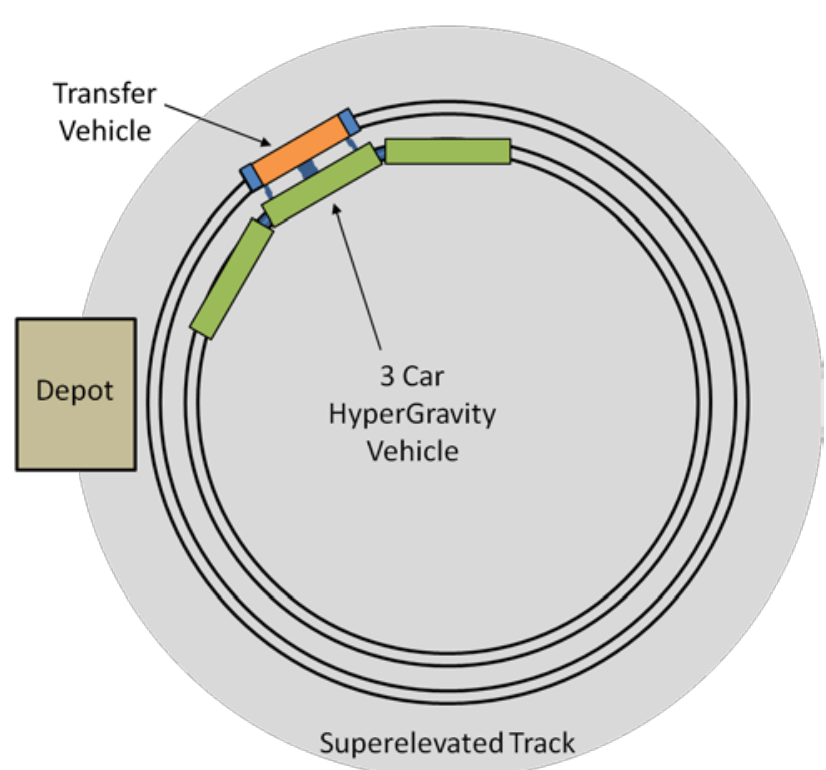

Figure 6. ESHGF System Top View depicted in a 3-car HGV configuration (figure not to scale)

\section{B. HyperGravity Vehicle Subsystem}

HGV subsystem is an electric train consisting of a sequence of connected cars running on a track. The top view of this subsystem is shown in Fig. 6 and its elements and their interactions are depicted in Fig. 8. People will be able to move between cars as they can onboard a typical train.

The general design is for all HGV Cars (HGVC)s to be the same size, but custom-size cars can be accommodated. 
The interior of each car is expected to be customized to meet the needs of its occupants and manager. Car types are expected to include at a minimum living quarters and workspace. Other car types may include dining facilities, farms, aquariums, pools, arboretums, botanic gardens, factories, gyms, sports center, markets, meeting rooms, etc.

The three major elements of each HGVC: Cabin, Chassis, and Cabin Tilt Mechanism, are depicted in Figs. 9 and 10.

An HGVC with a $24 \times 4 \times 4 m$ cabin on a $300 \mathrm{~m}$ diameter $30^{\circ}$ superelevated (banked) track with an additional Cabin Pivot Angle of $30^{\circ}$ is shown in perspective in Fig. 11

As per the third highlighted row in Table 1 , to be discussed in section II.F.1, the HGVC depicted in Fig. 11 is operating on the track at 3.2 RPM and generating 1.97g. At a lower RPM, the Cabin Pivot Angle would be less with a corresponding lower hypergravity level. HGCV stability design considerations to compensate for such a steeply superelevated track and HGVCs with high pivot angles are also discussed.

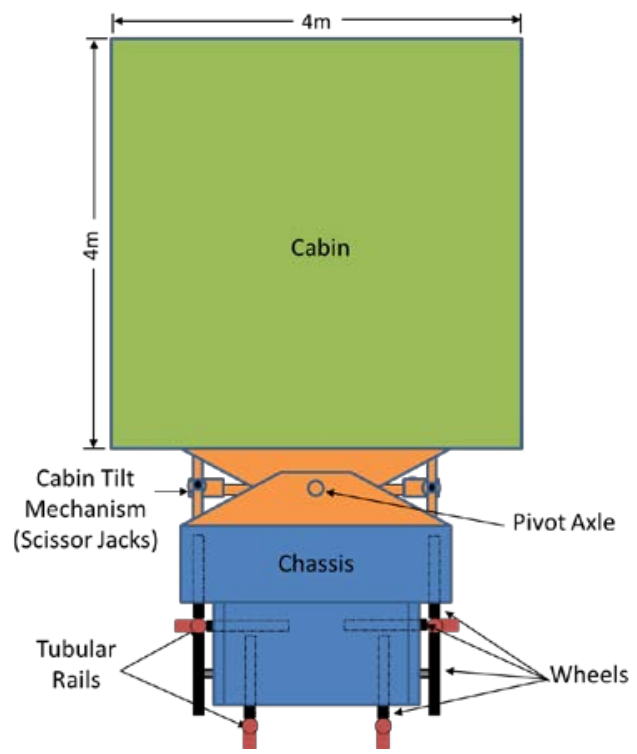

Figure 9. HGVC Front View (same as Rear View)

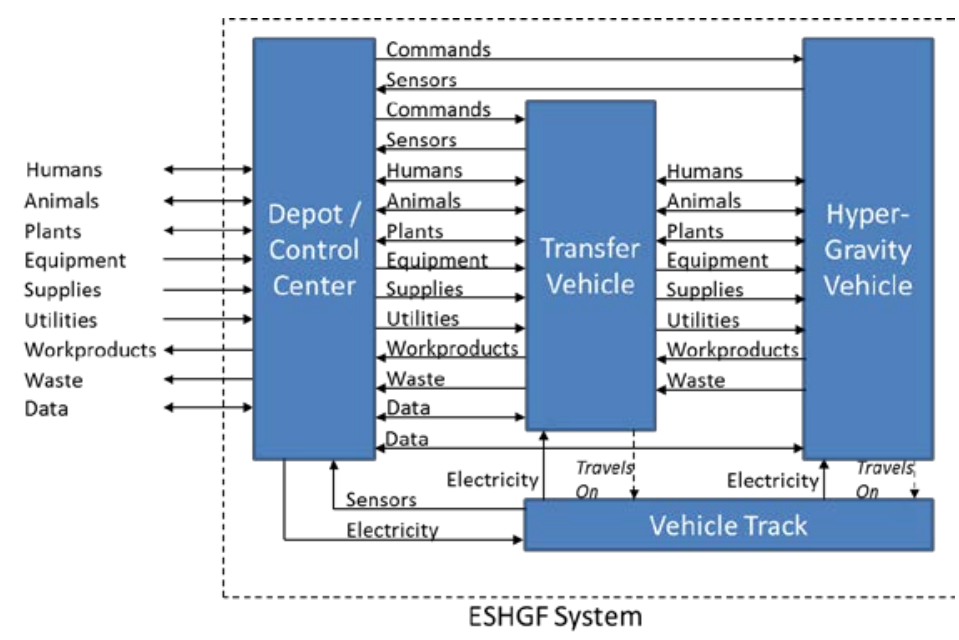

Figure 7. Svstem Overview

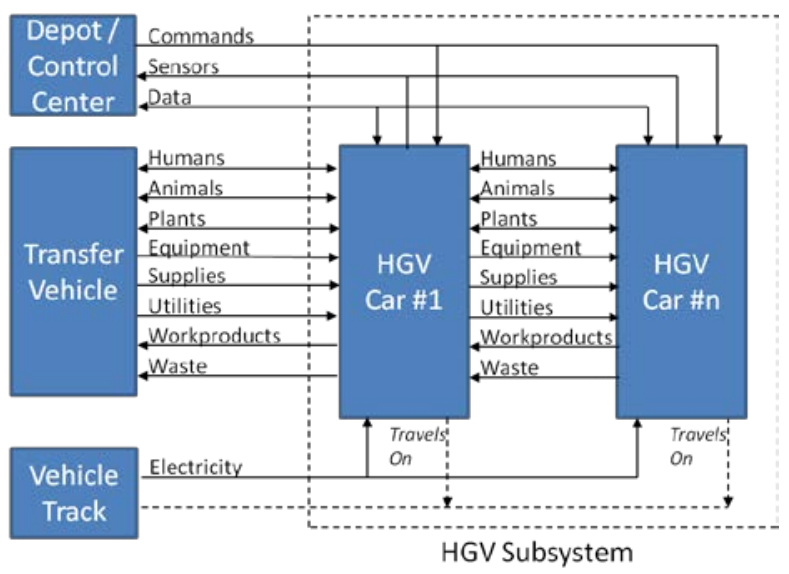

Figure 8. HGV Subsystem

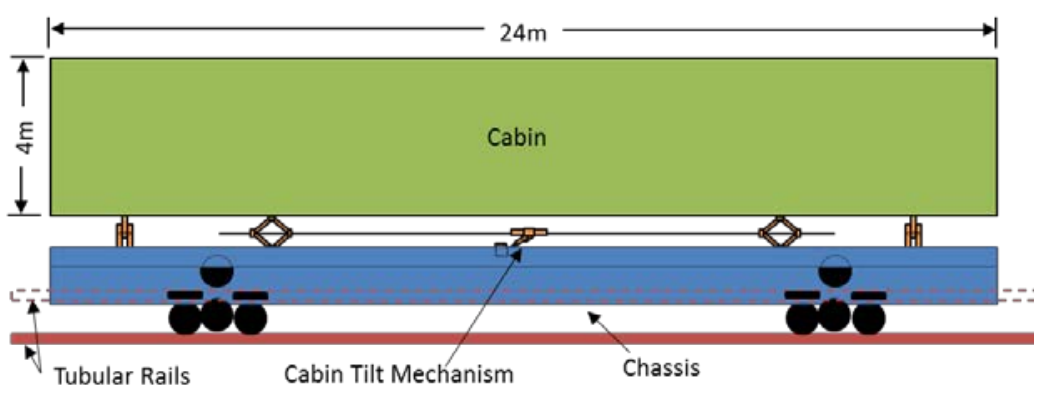

Figure 10. HGVC Side View

(the same for both sides)

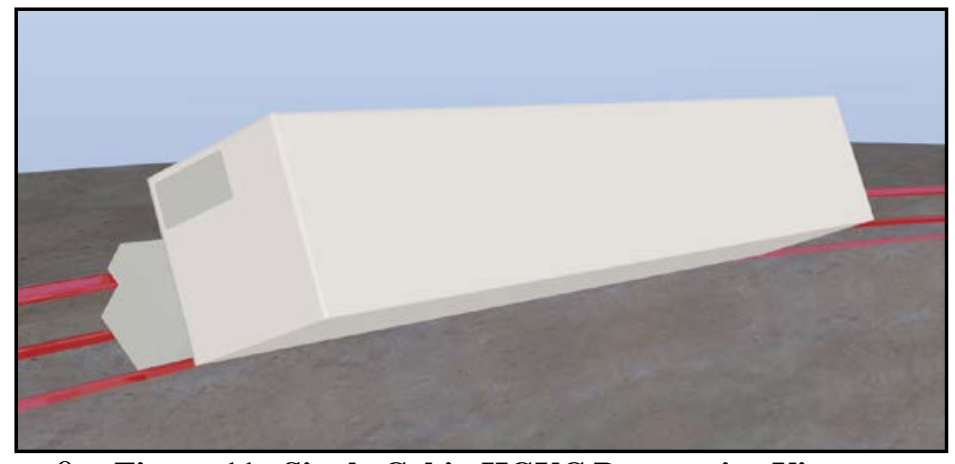

9 Figure 11. Single Cabin HGVC Perspective View

American Institute of Aeronautics and Astronautics 


\section{HGVC Chassis Subsystem}

The chassis is similar to a flatbed train car. It basically consists of a frame resting on two train wheel trucks, powered by electric traction motors, which run on a tubular rail track (see Fig. 15). Variations of the chassis include those without motors requiring another car to provide locomotion. Ideally, the chassis would be magnetically levitated (maglev) to operate efficiently at high speeds as well as to reduce maintenance, friction, noise, and vibration, but it is not necessary and the additional costs and development delays may not be justified.

\section{HGVC Cabin Subsystem}

The nominal cabin sizes are $4 m$ wide, $4 m$ high, and either $12 m$ or $24 m$ long. These sizes were selected due to their similarity to train car sizes and their feasibility as analogues for offEarth use. A space-rated version of this cabin could be accommodated as a payload on a heavy-lift launch vehicle for use on orbit, in the Moon, in Mars, in the Mars' moons. and in

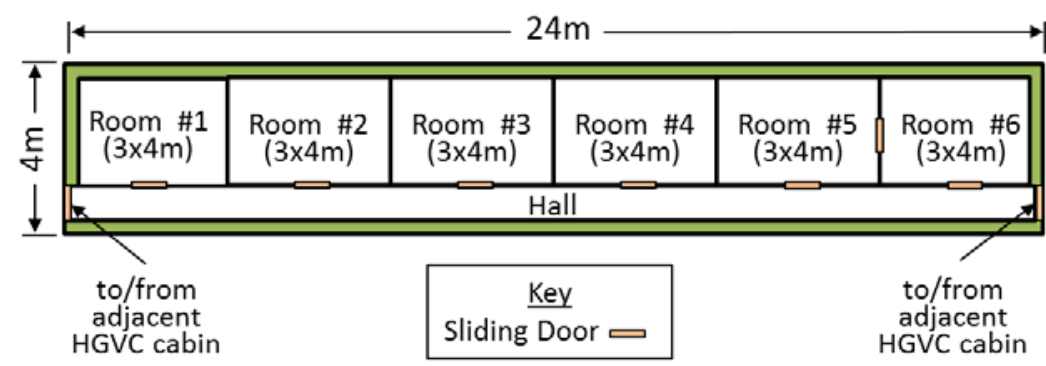

Figure 12. HGVC 6-room Cabin Floor Plan Example (Top View) asteroids. By taking space-rated cabin requirements into consideration, the ESHGF can be used as an analogue for such modular artificial-gravity space facilities and validating their requirements.

As shown in Fig. 12, each HGVC has a cabin with a hall that can connect to an adjacent HGVC cabin for people and cargo to travel the length of the entire HGV. This figure depicts a 6-room floor plan. However, the floor plans are flexible because internal cabin walls are not load bearing and may be moved.

\section{HGVC Tilt Mechanism Subsystem}

A special feature of each HGVC is its capability to control the tilt of its cabin so that it is relatively level with respect to its hypergravity vector. The HGCV tilt mechanism subsystem enables this capability by actively controlling the tilt of the cabin with respect to the chassis depending on the velocity of the HGVC. Views of the HGVC Cabin Tilt Mechanism Subsystem views are shown in Figs. 9, 13, and 14.

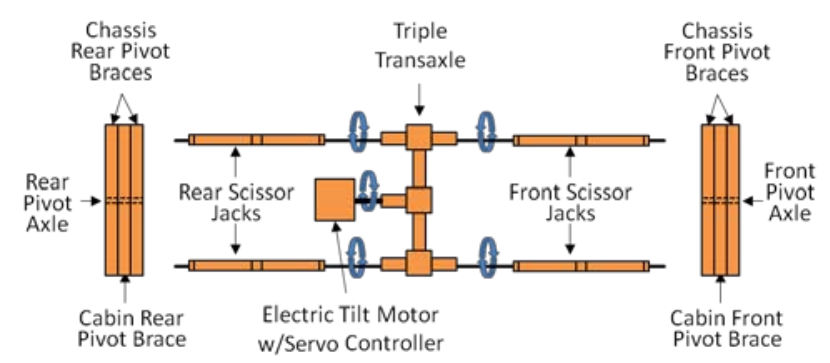

Figure 13. HGVC Tilt Mechanism Subsystem Top View

(attached between chassis and cabin on each HGVC; blue arrows show rotations when pivoting to the right)

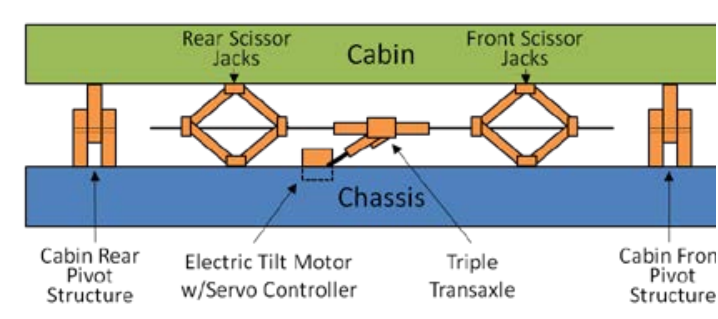

Figure 14. HGVC Cabin Tilt Mechanism Subsystem Side View

(both side views are the same)

As shown in these views, the HGVC Cabin Tilt Mechanism attaches the HGVC cabin and chassis by means of two pivot structures and four scissor jacks. The scissor jacks are used to change the tilt of the cabin with respect to the chassis. A triple transaxle is used to transfer the input torque from the electric tilt servo motor to the four threaded rods of the scissor jacks. The triple transaxle outputs torques such that as the left jacks are raised, the right jacks are simultaneously lowered as shown in Fig. 13. The electric tilt motor and its servo controller precisely control tilting the cabin to the commanded angle. The commanded angle is a function of the HGV velocity and the track (chassis) superelevation angle defined by Eq. (3).

The design can accommodate additional jacks if needed and other types of cabin tilt mechanisms can be used instead, e.g., hydraulic jacks, but scissor jacks have the advantage of a highly controlled relationship between the 
jack height and tilt degree as well as lower maintenance requirements. A design alternative for the HGV velocity input to the servo controller is an inclinometer-signal input that the servo controller zeros by tilting the cabin.

\section{HGVC Chassis Truck Subsystem}

The cabin(s) and its tilt mechanism rest on the chassis, which is used to move the car on the track discussed in section II.C. The chassis consists of a frame attached to two electric-traction motor trucks. Each truck rides on the track and can passively rotate with respect to the

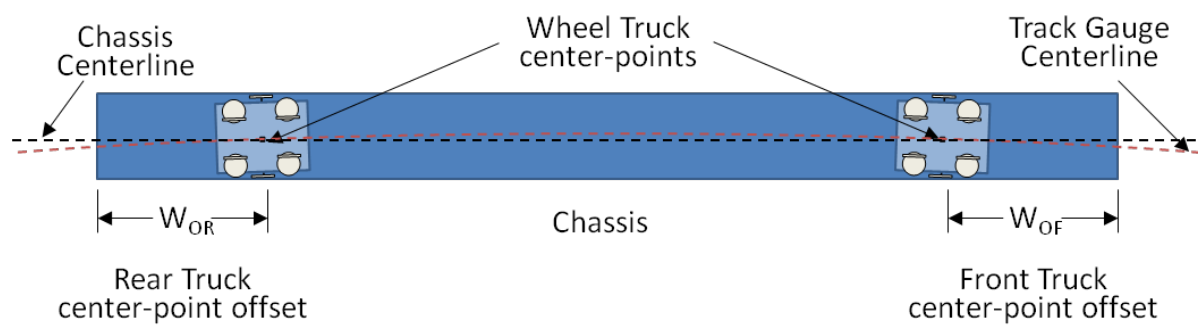

Figure 15. HGVC Chassis Bottom View chassis in order to stay centered on tracks. Although truck wheels may also passively rotate in response to another car pushing or pulling the chassis, in this design each truck is powered by electric motors to increase the locomotive traction and provide redundancy. The trucks are shown in Fig. 15, the HGVC chassis bottom view.

The two truck center-points on the chassis are the only two points on the chassis lengthwise centerline that are exactly the track radius distance (measured from the track circle center to the upper track gauge centerline - see Fig. 32). The front truck offset $\left(W_{O F}\right)$ and rear truck offset $\left(W_{O R}\right)$, shown in Fig. 15, are equal to facilitate attaching HGVCs to each other and denoted by the single parameter $\left(W_{T O}\right)$, i.e., $\left(W_{T O}=W_{O F}=W_{O R}\right)$. The value of $W_{T O}$ is significant with respect to hypergravity variations to be discussed in section II.F and the chassis centerline center offset $\left(r_{c o}\right)$ per Eq. (7).

\section{HGVC Stability Design Considerations}

The primary HGVC stability issue that must be addressed is atypical for trains and is due to the high side loads, and in some cases negative loads, on the HGVC wheels and tracks which could lead to an HGVC derailing and tipping over if not engineered properly. These loads are due to the steep track superelevation angle $\left(30^{\circ}\right)$, the tight turn radius $(150 \mathrm{~m})$, the high speeds $(112 \mathrm{mph})$, the pivoting cabins $\left(+-30^{\circ}\right)$, the shifting cargo and passengers, and the high cabin surface area subject to winds (if the ESHGF is not enclosed). In this design, the risk of derailment is mitigated by the HGVC horizontal and vertical wheels on the chassis wheel trucks that ride on three sides of the tubular-track side rails as shown in Figs. 9, 10, 11, and 15.

\section{Inter-HGVC Connectivity}

Initial HGV testing can be accomplished with a single HGVC. This design concept supports a multiple, connected HGVs that operate on the same track. These connections may be accomplished by extending the HGVC chassis and using train chassis couplers and the same way that most train cars are connected, but other methods may perform better. Standard train chassis couplers do not take advantage of the fact that the HGV runs on a superelevated, level track with a constant radius. Depending on the precision of the track, the HGVCs may be connected by a variety of other methods that provide additional strength, rigidity, and vibration damping. The details of how the HGVCs are connected require further study and are beyond the scope of this document.

In addition to the chassis connector, HGVs with multiple HGVCs also require flexible vestibules that provide a passageway between cabins on connected HGVCs similar to those between passenger train cars, but in this case the vestibules tilt with the cabins so the vestibule floor also is effectively level regardless of the HGV velocity. These vestibules are dependent on the HGVCs coupling design and further details are also beyond the scope of this document.

\section{Vehicle Track Subsystem}

The Vehicle Track subsystem is the pathway for the HGV and Transfer Vehicle and provides electricity to both subsystems. Both tracks are superelevated and circular as depicted in Fig. 16. Side views of the superelevated track are shown in Figs. 18 and 20. As depicted in both figures, a shell may be used to cover the track, HGV, and Transfer Vehicle to protect them from the weather and provide security. 
Each track has four tubular rails (two bottom rails and two side rails as shown in Figs. 18 and 19) to carry the load and provide stability for the vehicle cars. An additional rail (not shown) provides power to the cars as is typical for the 3rd rail for electric trains. The key track requirements are that the track be circular (track radius is constant), level (perpendicular to the gravity vector), and have a constant superelevation angle with respect to the gravity vector. The track foundation is implemented with a reinforced steel and concrete ring to minimize track deformation over time, but could also be implemented in a bedrock tunnel.

An optional augmentation to the track design concept presented is for the tracks to support maglev cars to minimize their energy use, noise, vibration, and wear. At low speeds and in emergency cases the vehicle wheels would contact the tracks, but during nominal operations the vehicles do not contact the tracks other than for power. Once the HGV reaches a significant operating speed, the passengers will not easily detect that the cars are moving. However, they will be able to detect their bodyweights and cabin content weights have increased.

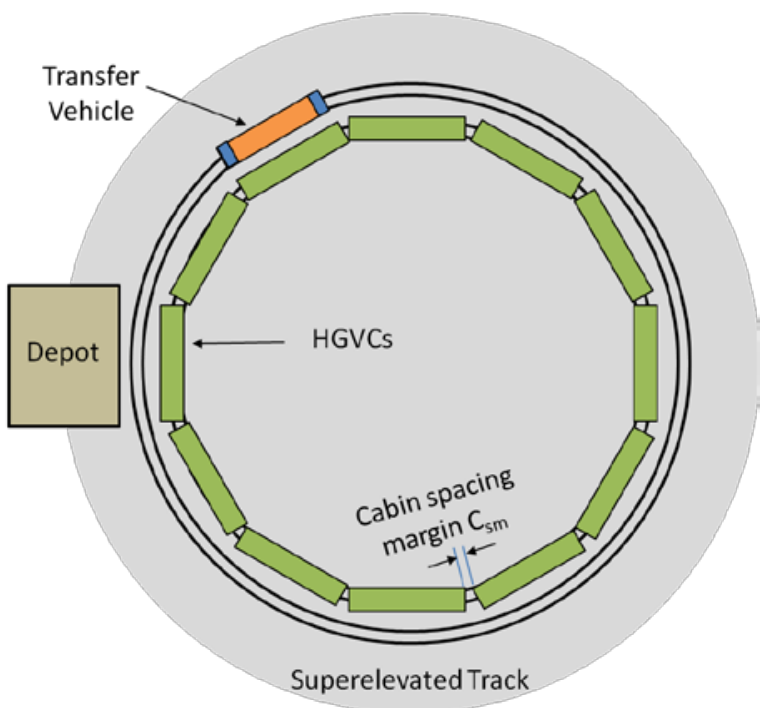

Figure 16. ESHGF System depicted in a HGV complete ring configuration (figure not to scale)

Fig. 16 illustrates the ESHGF completely filled with HGVCs and the Transfer Vehicle (not to scale). However, the ESGV can easily be incrementally completed and operated in a minimal configuration of a single HGVC without the Transfer Vehicle.

The HGVCs must be spaced with sufficient margin between cabins to prevent contact between cabins when tilted. This spacing is discussed further in the appendix.

\section{Transfer Vehicle Subsystem}

The top view of a Transfer Vehicle aligned with a multi-car HGV is shown in Fig. 16. The purpose of the Transfer Vehicle is to transfer people and cargo between the Depot and the HGV while the HGV is running so that the HGV can indefinitely maintain a constant velocity and minimize disruptive hypergravity-level changes. In addition, using a Transfer Vehicle reduces the energy required to operate the ESHGF since the entire HGV, which can be several times the mass of the Transfer Vehicle, does not have to stop and restart every time someone or something needs to go on or come off the HGV. The Transfer Vehicle subsystem is similar in design to a single-car HGV, as shown in Figs. 9 and 10, but may be shorter depending on the transfer capacity required. Also, the Transfer Vehicle may be augmented with a cabin elevator as shown in Figs. 18 and 19.

The Transfer Vehicle cabin is equipped with a Retractable Vestibule for ingress and egress with the HGV and two chassis Retractable Arm Locks for the Transfer Vehicle and HGV to maintain a constant relative position while docked to each other. At least one HGVC is equipped with two Retractable Arm Locks and a docking port for the Transfer Vehicle Retractable Vestibule as depicted in Figs. 17 and 18.

In this design concept, the Transfer Vehicle and its track either can be designed for docking with the HGV at its entire range

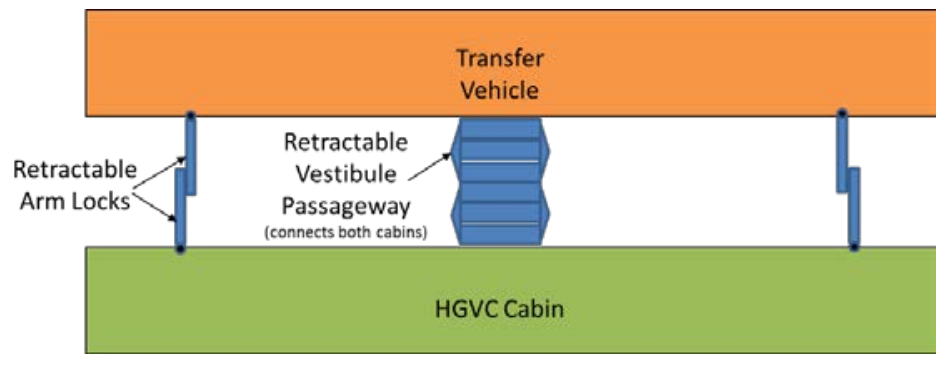

Figure 17. Transfer Vehicle temporarily connected to HGVC Top View of velocities and corresponding cabin pivot angles or for only docking at a specific HGV velocity and cabin pivot angle. In order for the Transfer Vehicle to dock with the HGV at a range of velocities, the Transfer Vehicle is required to elevate as well as tilt its cabin for docking. This is accomplished with a cabin elevator that raises and lowers the Transfer Vehicle cabin and its tilt mechanism as shown in Figs. 18 and 19. 

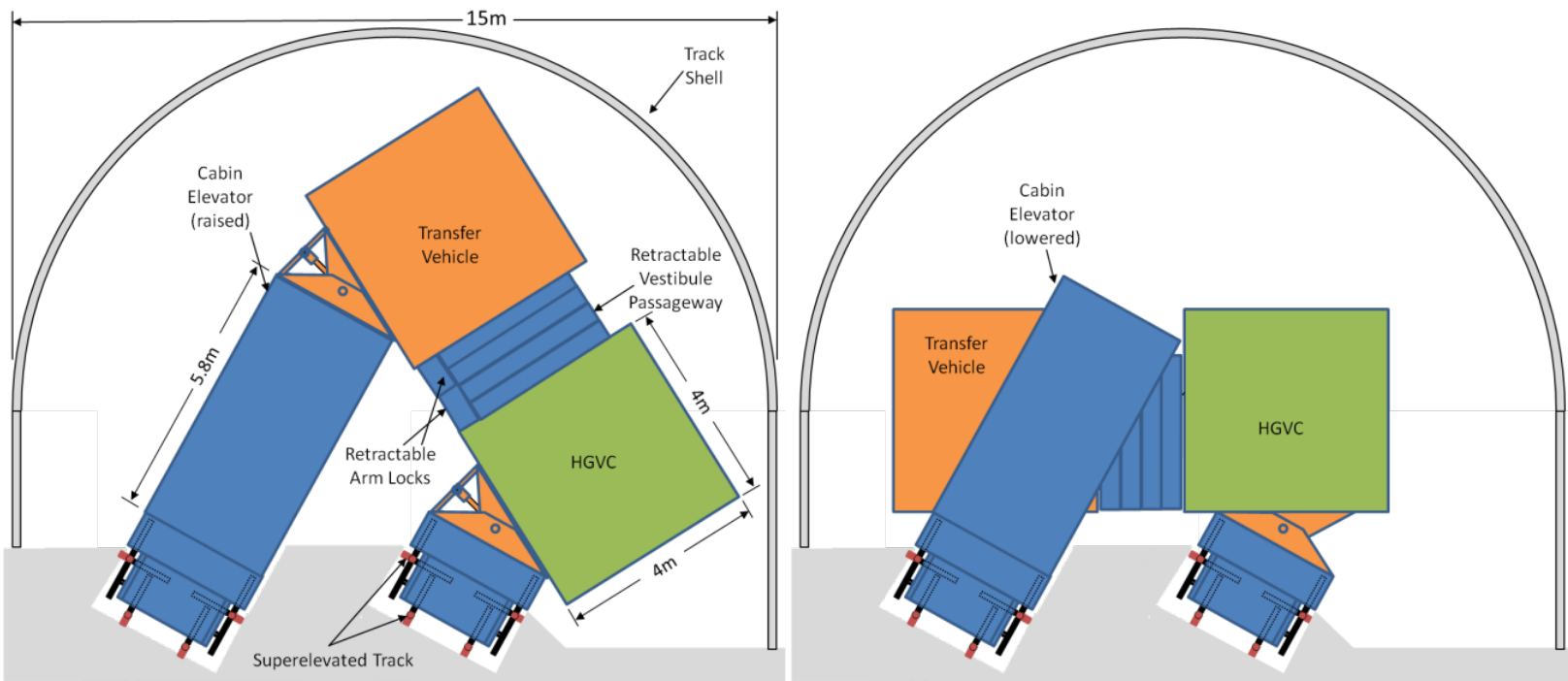

Figure 18. Transfer Vehicle Docking with HGVCs Rear Views in Max $g$ Orientation (left) and Stopped Orientation (right)

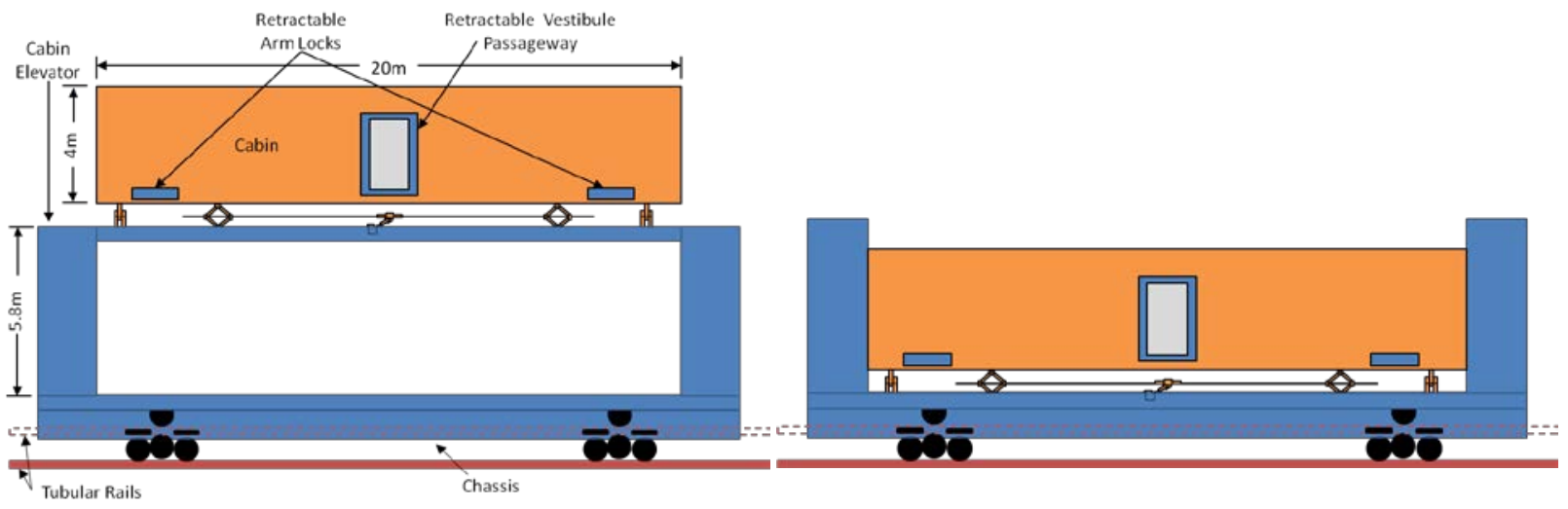

Figure 19. Transfer Vehicle Side Views

Cabin Elevator Raised (left) and Lowered (right)

However, the Transfer Vehicle is not required to elevate its cabin if it is designed only to dock when the HGV is at a specific operating speed and cabin pivot angle as shown in Fig. 20.

A design alternative to the Transfer Vehicle Retractable Vestibule is for the entire Transfer Vehicle cabin to translate horizontally and vertically to directly dock with an HGV cabin. An alternative to the entire Transfer Vehicle subsystem is the Hub \& Spoke subsystem discussed in section II.H.3. 


\section{E. Depot / Control Center Subsystem}

The Depot / Control Center (DCC) subsystem (see Fig. 16) controls the HGV and Transfer Vehicle and is similar in design and function to train stations and airport terminals. The DCC facilitates the ingress and egress of people, animals, plants, equipment, supplies, utilities, workproducts, waste, and data between the ESHGF and outside the system. The DCC also acts as a service station for the HGV and Transfer Vehicle.

\section{F. HGV HyperGravity}

\section{HGV HyperGravity Level}

The hypergravity level of the HGV is the vector sum of the ambient gravity vector at the HGV location and the perpendicular centripetal-acceleration vector created by the HGV travelling in a circle. The hypergravity level at a point in an HGVC cabin is a function of the track radius and HGVC velocity at that point and is defined by Eq. (1). The HGVC Cabin coordinate frame, depicted in Fig. 22) is defined with its origin at the center of the bottom of the cabin; the $+x$-axis runs lengthwise toward the cabin front; the $+y$-axis runs widthwise directly opposed to the track center; and the +z-axis runs up toward the cabin ceiling.

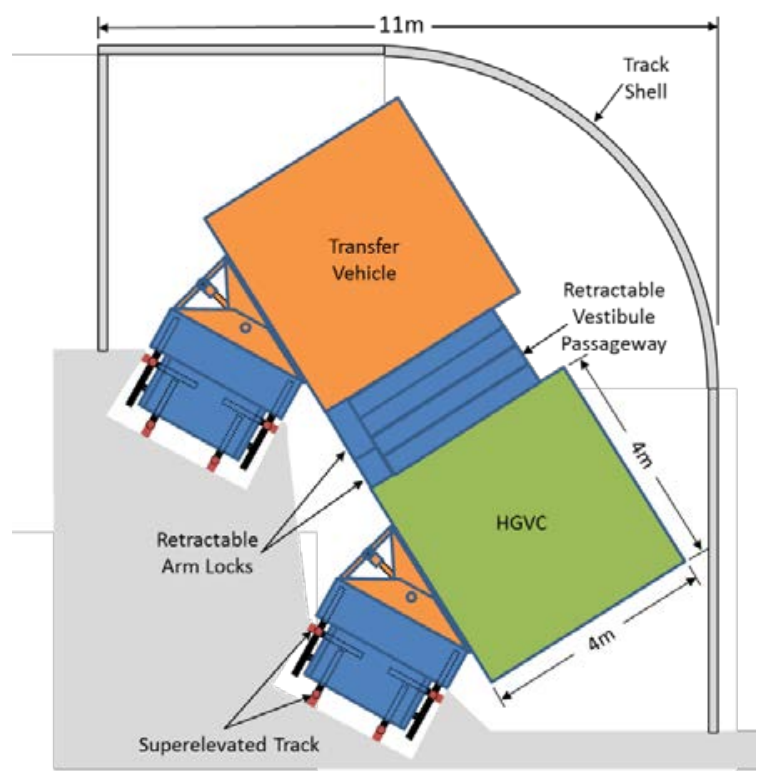

Figure 20. Transfer Vehicle temporarily connected to HGVC Rear View

$$
H_{\text {pos }}\left(x, y, z, P, v_{W}, g_{a}\right)=\frac{\sqrt{\mathrm{g}_{a}^{2}+\left(\frac{\mathrm{v}_{\text {pos }}\left(x, y, z, \mathrm{P}, \mathrm{v}_{\mathrm{W}}\right)^{2}}{r_{\text {pos }}(x, y, z, P)}\right)^{2}}}{9.807}
$$

The HGVC cabin coordinate frame changes with respect to its HGVC coordinate frame by the cabin pivot angle $P$, which changes the radii and velocities of the points in the cabin respectively according to Eq. (1). The cabin point velocities vary within the cabin per Eq. (2), but the overall HGVC velocity, $v_{W}$, is controlled by the HGVC wheel truck.

$$
v_{p o s}\left(x, y, z, P, v_{W}\right)=\frac{v_{w} \cdot r_{p o s}(x, y, z, P)}{r_{T}}
$$

Note that changing the Cabin Pivot Axle Angle $P$ changes the radii and velocities of the points in the HGVC cabin even though the HGVC RPM on the track remains constant. Hence, track radius $r_{T}$ is a rough approximation of the actual radius of an HGVC cabin point, $r_{p o s}(x, y, z, P)$, defined by Eq. (4); and consequently the HGVC wheel truck velocity $v_{W}$ is a rough approximation of the HGVC cabin point velocity, $v_{\text {pos }}\left(x, y, z, P, v_{W}\right)$. These approximations do not hold for cases in which the track radius $r_{T}$ is small relative to the cabin width $\mathrm{y}$ and height $\mathrm{z}$, such as for an extreme case in which tilting the cabin places the track circle center underneath cabin points (the velocity is 0 for cabin points directly over the track circle center regardless of the HGVC RPM).

Eq. (1) is derived from the equation defining centripetal acceleration $=$ velocity $2 /$ radius, and the equation for adding two perpendicular vectors (Pythagorean Theorem). Note that the centripetal acceleration (hypergravity excluding the ambient gravity) is inversely proportional to the track radius, but is directly proportional to the square of the HGV velocity. For example, halving the track radius doubles the hypergravity increase due to centripetal acceleration; but doubling the HGV velocity quadruples the gravity due to centripetal acceleration. Table 1 provides more detailed examples.

Note that the $g$ 's calculated in Table 1 do not take into account small variances due to HGV dimensions, such as the cabin floor height, width, and length, to be discussed in section II.F.3. 
Table 1. Hypergravity Level Examples

\begin{tabular}{|c|c|c|c|c|c|c|c|c|c|}
\hline Location & $\begin{array}{c}\text { HGV } \\
\text { Ambient } \\
\text { (Location) } \\
\text { Gravity } \\
\left(\mathrm{m} / \mathrm{s}^{2}\right) \\
\end{array}$ & $\begin{array}{c}\text { Track } \\
\text { Dia } \\
(m) \\
\end{array}$ & $\begin{array}{c}\text { HGV } \\
\text { Velocity } \\
(m / s) \\
\end{array}$ & $\begin{array}{c}\text { HGV } \\
\text { mph }\end{array}$ & $\begin{array}{l}\text { HGV } \\
\text { RPM }\end{array}$ & $\begin{array}{c}\text { Cabin } \\
\text { Pivot } \\
\text { Angle } \\
\text { (deg) } \\
\end{array}$ & $\begin{array}{c}\text { Track } \\
\text { Super- } \\
\text { elevation } \\
\text { (deg) }\end{array}$ & $\begin{array}{c}\text { Centripetal } \\
\text { Acceleration } \\
\text { (Horizontal) } \\
\text { g's } \\
\end{array}$ & $\begin{array}{l}\text { HGV } \\
\text { Hyper- } \\
\text { gravity } \\
\text { g's } \\
\text { (H) } \\
\end{array}$ \\
\hline Earth & 9.807 & 100 & 0 & 0 & 0.0 & -30 & 30 & 0.00 & 1.00 \\
\hline Earth & 9.807 & 100 & 10 & 22 & 1.9 & -18 & 30 & 0.20 & 1.02 \\
\hline Earth & 9.807 & 100 & 20 & 45 & 3.8 & 9 & 30 & 0.82 & 1.29 \\
\hline Earth & 9.807 & 100 & 35 & 78 & 6.7 & 38 & 30 & 2.50 & 2.69 \\
\hline Earth & 9.807 & 250 & 25 & 56 & 1.9 & -3 & 30 & 0.51 & 1.12 \\
\hline Earth & 9.807 & 250 & 35 & 78 & 2.7 & 15 & 30 & 1.00 & 1.41 \\
\hline Earth & 9.807 & 250 & 50 & 112 & 3.8 & 34 & 30 & 2.04 & 2.27 \\
\hline Earth & 9.807 & 300 & 25 & 56 & 1.6 & -7 & 30 & 0.42 & 1.09 \\
\hline Earth & 9.807 & 300 & 40 & 89 & 2.5 & 17 & 30 & 1.09 & 1.48 \\
\hline Earth & 9.807 & 300 & 50 & 112 & 3.2 & 30 & 30 & 1.70 & 1.97 \\
\hline Earth & 9.807 & 500 & 25 & 56 & 1.0 & -16 & 30 & 0.25 & 1.03 \\
\hline Earth & 9.807 & 500 & 50 & 112 & 1.9 & 16 & 30 & 1.02 & 1.43 \\
\hline Earth & 9.807 & 500 & 75 & 168 & 2.9 & 36 & 30 & 2.29 & 2.50 \\
\hline Mars & 3.711 & 250 & 34 & 76 & 2.6 & 33 & 35 & 0.94 & 1.02 \\
\hline Mars & 3.711 & 300 & 37 & 83 & 2.4 & 33 & 35 & 0.93 & 1.00 \\
\hline Mars & 3.711 & 500 & 48 & 107 & 1.8 & 33 & 35 & 0.94 & 1.01 \\
\hline Moon & 1.622 & 250 & 35 & 78 & 2.7 & 41 & 40 & 1.00 & 1.01 \\
\hline Moon & 1.622 & 300 & 38 & 85 & 2.4 & 40 & 40 & 0.98 & 1.00 \\
\hline Moon & 1.622 & 500 & 50 & 112 & 1.9 & 41 & 40 & 1.02 & 1.03 \\
\hline On-orbit & 0 & 250 & 35 & 78 & 2.7 & 0 & 90 & 1.00 & 1.00 \\
\hline On-orbit & 0 & 300 & 38 & 85 & 2.4 & 0 & 90 & 0.98 & 0.98 \\
\hline On-orbit & 0 & 500 & 50 & 112 & 1.9 & 0 & 90 & 1.02 & 1.02 \\
\hline
\end{tabular}

Table 1 captures a number of the trades in order to obtain the desired hypergravity level. For a given hypergravity level, the required HGV velocity can be reduced by decreasing the track diameter per Eq. (1), but this also increases the HGV RPM. Large track diameters increase costs and high HGV velocities increase risks, but high RPMs increase the likelihood and intensity of motion sickness and possible long-term side effects. The long-term effects of rotation on humans is not well understood (excluding one revolution per day), but rates above 2-3 RPMs are known to cause motion sickness in a high percentage of the population when not trained or treated accordingly. The capability to study these long-term effects is one of the benefits of the ESHGF.

Additional examples are given at various HGV velocities with four different track diameters $(100 \mathrm{~m}, 250 \mathrm{~m}$, $300 \mathrm{~m}$, and $500 \mathrm{~m}$ ), at four different locations (Earth, Mars, Moon, and on-orbit) for considering the ESHGF as an analogue for off-Earth artificial-gravity facilities. The on-orbit example rows are provided since such an ESHGF would be similar to an orbiting space station in a wheel configuration with spokes and connected rim modules instead of tracks and HGV cars. The on-orbit examples are also similar to ESHGFs on an asteroid or a Mars moon. Note that once the ambient gravity of the ESHGF is reduced to the gravity level of the Moon $\left(1.622 \mathrm{~m} / \mathrm{s}^{2}\right)$, the ambient gravity has little effect on the hypergravity level $(H)$ where the centripetal acceleration is several times higher than the ambient gravity (as shown in Table 1).

The optimal track diameter of $300 \mathrm{~m}$ for this design concept is shown by the highlighted rows in Table 1 . A design with a hypergravity level of up to $2 g$ 's is required to provide the capability to generate a wide range of hypergravity levels humans can live at for long periods. Smaller track diameters require RPM levels that are too high for a $2 g$ hypergravity level. For larger diameters for a $2 g$ hypergravity level, the HGV velocities are too high as well as being more costly. An HGV configured as the third highlighted row is shown in perspective in Fig. 11. For this example, the track is $300 \mathrm{~m}$ diameter with a $30^{\circ}$ superelevation and a Cabin Pivot Angle of an additional $30^{\circ}$. This HGV has a hypergravity of $1.97 \mathrm{~g}$ when running $50 \mathrm{~m} / \mathrm{s}(112 \mathrm{mph})$, which is $3.2 \mathrm{RPMs}$. The $300 \mathrm{~m}$ diameter is also a reasonable size for an orbiting space settlement, which would generate $1 \mathrm{~g}$ at 2.4 RPMs.

An ESHGF can be designed with multiple concentric tracks of different diameters to support simultaneously generating multiple levels of hypergravity. Multi-track ESHGF designs are discussed in section II.H. 


\section{HGV Hypergravity Control}

The hypergravity is controlled by controlling the HGV velocity per Eq. (1). Consequently, the hypergravity can be kept constant by maintaining a constant HGV velocity.

In addition to controlling the hypergravity level of the HGV, the HGV controls the cabin tilt angle per Eq. (3) so that regardless of the HGV velocity, the down direction (hypergravity vector) is always perpendicular to the cabin floor at its center as shown in Fig. 21. The Cabin Pivot Angle specified in Table 1 is measured with the vertex at the center of the HGV pivot such that at a Cabin Pivot Angle of 0 degrees, the cabin floor is parallel with the chassis bed and track superelevation. The sum of the Cabin Pivot Angle and the Track superelevation angle is the angle of the cabin floor to the track circle plane, which is perpendicular to the ambient gravity vector, i.e., level.

For example, consider the first example in Table 1 . The HGV is stopped on a track with a $30^{\circ}$ superelevation. The Cabin Pivot Angle is $-30^{\circ}$ in order to maintain the down direction perpendicular to the cabin floor. This case is illustrated in Fig. 21 left.
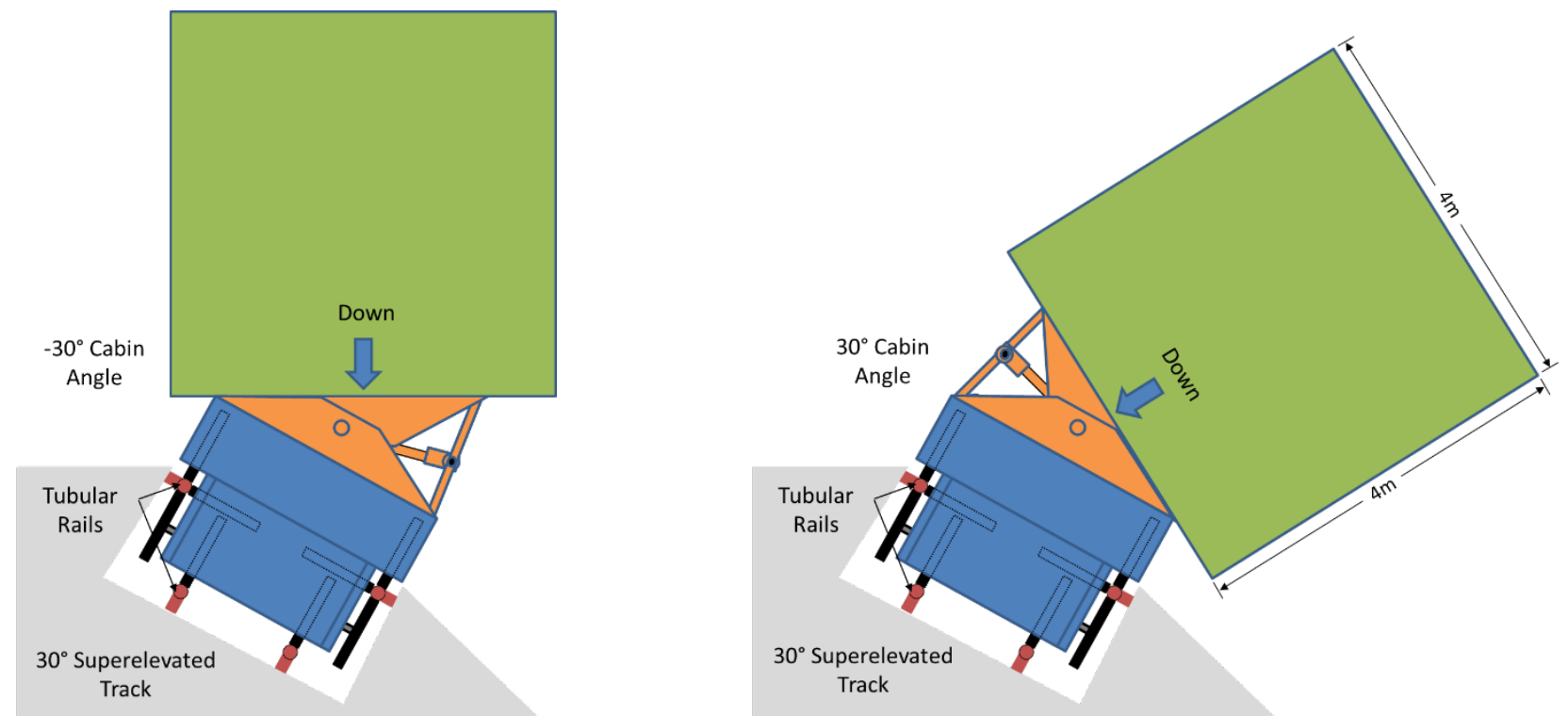

Figure 21. HGV Car in Stopped Orientation (left) and Max g Orientation (right)

As the HGV velocity increases, the Cabin Pivot Angle increase until it reaches its maximum value of $30^{\circ}$ as depicted in Fig. 21 right.

With a sufficiently slow HGV acceleration rate and Cabin Pivot Angle change rate, neither change will be noticed by the occupants. For example, a glass of water resting on a table would appear to remain unchanged as the HGV accelerates and then maintains its operational velocity. The only difference with regard to the glass of water is that it would become heavier.

In order to maintain a constant down direction at any velocity, the tilt command input is maintained at the calculated cabin tilt angle, which is a function of the HGV velocity and is defined by Eq. (3).

$$
P=\tan ^{-1}\left(\frac{\mathrm{v}_{\mathrm{w}}^{2}}{\mathrm{r}_{\mathrm{T}} \cdot \mathrm{g}_{\mathrm{a}}}\right) \cdot \frac{180}{\pi}-T_{B}
$$

The Track Circle Radius Bias, $r_{\text {bias }}$, is a HGVC tunable parameter, nominally between 0 and 2, that is used to fine-tune the cabin pivot angle since the hypergravity level does vary slightly throughout the cabin as described in the next subsection.

\section{HGVC Cabin Gravity Map}

Ideally, the hypergravity level would be equal and constant for all points in a HGVC cabin for the gravity to behave more like the way people are accustomed to on Earth and for consistency in experiments. However, there will be minor gravity variations at different points in the cabin that are different distances from the center of the HGV track circle and consequently have different centripetal accelerations and corresponding hypergravity levels. 
The advantage of using a large radius track is that these variances are minimal. In an extreme case where the track circle is so small that the track radius is zero for points in a HGVC cabin, the centripetal acceleration for an object centered at such a point would also be zero.

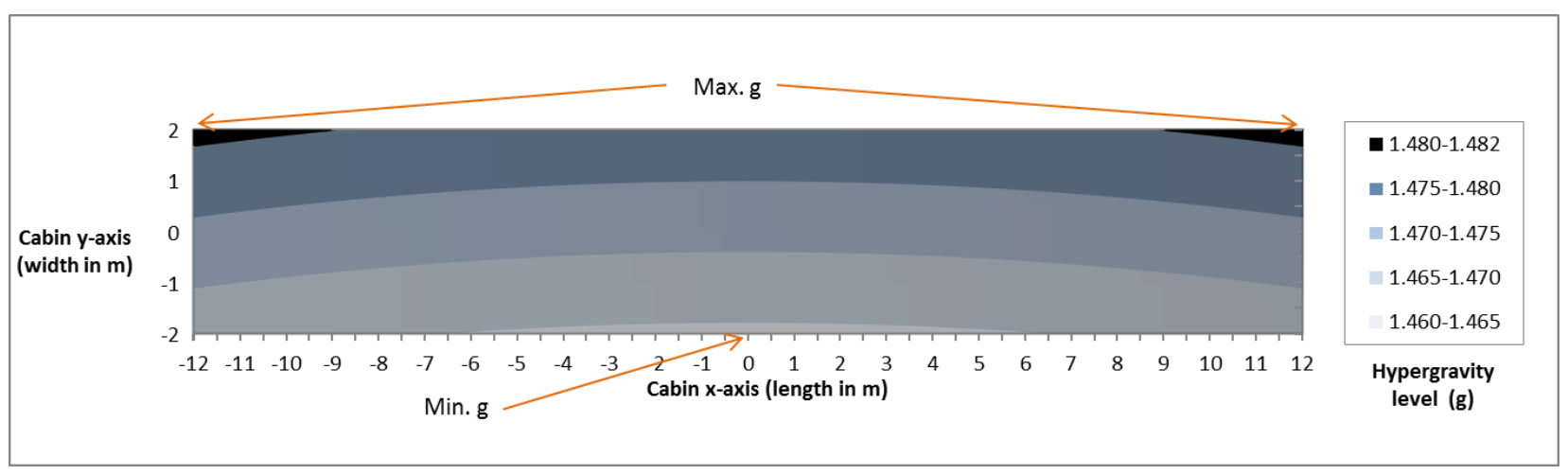

Figure 22. HGVC 24 x 4m Floor Hypergravity Map Example

An example of an HGVC cabin hypergravity floor map illustrating microgravity variations is shown in Fig. 22. Pertinent parameters for this example are the HGVC cabin coordinates (x, y), the Cabin Pivot Angle $=17.6^{\circ}$, the track radius $=150 \mathrm{~m}$, the HGV velocity $=40 \mathrm{~m} / \mathrm{s}$, track superelevation angle $=30^{\circ}$, Cabin Pivot Center height $=1 \mathrm{~m}$, Cabin floor height from pivot axle center $=0.5 \mathrm{~m}$, Cabin length $=24 \mathrm{~m}$, Cabin width $=4 \mathrm{~m}$, front $\&$ rear wheel truck offset $=3 \mathrm{~m}$, and ambient gravity $=9.807 \mathrm{~m} / \mathrm{s}^{2}$ (Earth); the relevance of these parameters are discussed below.

This hypergravity map varies with different HGV configurations and velocities, but in most cases the basic characteristics are similar. The hypergravity varies with the $(x, y, z)$ position of an object in the HGV cabin. The lowest-hypergravity point on the cabin floor is at the middle of the cabin where the floor meets the inner wall, i.e., position $(0,-2)$. The hypergravity at this position for this example is $1.464 \mathrm{~g}$ 's, so a $1 \mathrm{~kg}$ mass at this position would weigh $1.464 \mathrm{~kg}$. However, the hypergravity at both outer corners, positions $(-12,2) \&(12,2)$, is $1.481 \mathrm{~g}$ 's, so a $1 \mathrm{~kg}$ mass at both of those locations would weigh $1.481 \mathrm{~kg}, 17$ grams more, an increase of $\sim 1 \%$. In most cases, a variation of $1 \%$ would not be noticeable, but the hypergravity map is a design consideration. This variation increases considerably on smaller diameter tracks. Although the floor is flat, water would tend to flow and balls would tend to roll away from the inside center to the outside corners of the cabins, with an asymmetric bias toward the rear outside corner since any object moving away from the track circle center will gain momentum in the process. This variance is less within each cabin room since they are smaller.

The length, width, and height of the HGVC cabin all impact these hypergravity variations. A person moving directly across a cabin from its outside wall to its inside wall also moves further toward the center of the track circle and reduces the person's rotation radius. By increasing the sum of the track superelevation angle and cabin pitch angle to $90^{\circ}$ such that the cabin floor is perpendicular to the track plane, a change in width position will no longer cause a change in hypergravity. However, such a configuration is only appropriate in a low-gravity environment such as an on-orbit or on an asteroid. Reducing the width of the HGVC cabin will also reduce this variance, but this also can be accomplished by creating narrower rooms within the HGVCs as needed.

Since the cabins are straight instead of arced with the same radius as the track, moving toward the lengthwise center of a cabin also moves further toward the center of the track and reduces the spin radius (each cabin lengthwise centerline is a chord of the track circle - see Figs. 15 and 33). This variance can be mitigated by arcing the floor and shortening the HGVC cabin length or cabin room lengths if necessary.

The hypergravity level of an object decreases as it is raised in the cabin, e.g., an object on a table in the cabin will weigh less than the same object on the floor directly below it (with respect to the Cabin coordinate frame). Since the cabin is tilted toward the track circle center, increasing the height of an object decreases its track circle radius and its corresponding centripetal acceleration.

\section{G. ESHGF Modularity}

A key feature of the ESHGF is its modularity. Each module, comparable to intermodal shipping containers, can be transported by rail, truck, ship, and aircraft as shipping containers currently are transported. The size of these modules are also feasible for heavy lift launch vehicles. If necessary, the ESHGF Cars and/or Modular Cabins can 
be separated, transported, and reattached at their destination. Also, ESHGF cabins and cars can be added as needed to increase system capacity, as well as be removed for maintenance, to be upgraded, or replaced with modules tailored for different purposes.

\section{H. ESHGF System Capacity and Extensibility}

Significant artificial-gravity research can be accomplished with a single HGVC, but with the goal to ultimately create a scalable space habitat to support a permanent space community in excess of 1000 people, the ESHGF can be incrementally expanded to support such population-sizes as well as habitats for a wide variety of plants and animals.

The modular characteristic of the ESHGF design enables the system capacity to be incrementally increased as needed. The design presented focuses on a $300 \mathrm{~m}$ diameter track, but as discussed previously, other track sizes may be implemented. Capacity can be increased in a number of ways. In this section, the following methods to increase capacity are presented: increasing the number of cabins per HGVC, increasing the number of HGVCs per track, and increasing the number of concentric tracks.

\section{HGV Population Density}

The population density of the HGV can vary significantly based on the operations concept. An HGV could be operated unmanned as the lower bound of population density. An upper bound for consideration is provided by the U.S. Code of Federal Regulations Title 46 - Shipping which permits up to one person per each $0.9 \mathrm{~m}^{2}$ of deck area. ${ }^{58}$

However, a cruise ship may provide a more reasonable estimate for long-term population density. A population density analysis of a small sample of Royal Caribbean cruise ships ${ }^{59}$ is shown in Table 2.

Based on an average cruise ship population density of $28 \mathrm{~m}^{2}$ per person, a corresponding HGV population at the same density is 3.43 people per single cabin car. In the following section, multi-cabin cars are presented that increase the population up to 48 people per car, at the same density depending on the number of cabins added. A self-sufficient settlement with biomes would have a much lower population density.

Table 2. Cruise Ship Population Density Examples

\begin{tabular}{|l|c|c|c|c|}
\hline \multicolumn{1}{|c|}{ Ship Name } & $\begin{array}{c}\text { Max. Passengers } \\
\text { \& Crew }\end{array}$ & $\begin{array}{c}\text { Total Deck } \\
\text { Area, } \boldsymbol{m}^{\mathbf{2}}\end{array}$ & $\begin{array}{c}\text { Deck Area } \\
\text { per Person, } \boldsymbol{m}^{\mathbf{2}}\end{array}$ & $\begin{array}{c}\text { Ref } \\
\#\end{array}$ \\
\hline Adventure of the Seas & 5,020 & 137,000 & 27 & 60 \\
\hline Mariner of the Seas & 5,020 & 145,000 & 29 & 61 \\
\hline Navigator of the Seas & 5,364 & 137,000 & 26 & 62 \\
\hline Freedom of the Seas & 5,740 & 165,000 & 29 & 63 \\
\hline
\end{tabular}

\section{Multi-Cabin HGVCs}

Multiple cabins can be attached to a single HGVC to increase the HGV capacity and to generally reduce the path distance between two points in different cabins compared to cabins configured linearly on single-cabin HGVCs. A simple multi-cabin HGVC configuration has a second cabin on top of the first as illustrated in Fig. 23. This configuration doubles the HGVC deck area to $192 \mathrm{~m}^{2}$ (2,067 sq. ft.) without increasing the chassis size.

This figure also illustrates the ESHGF implemented in a circular tunnel, similar to a subway, instead of using a track shell. This is a more realistic analogue for an artificial-gravity facility on Mars, a moon, or an asteroid, which would benefit from the shielding and thermal insulation that being underground provides. If Tunnel Boring Machines $^{64,65}$ are used for mining at these locations, the tunnels they leave behind can be used for artificial-gravity settlements.

Once HGVCs with one or two cabins have been validated, HGVCs with many more cabins can be deployed by increasing the HGVC chassis width and track gauge as needed to accommodate the loads of the cabins and their contents. Additional rails can be added if needed as well.

Multiple views of a 26-Cabin HGVC configuration are shown in Figs. 24 and 25. In Fig. 24, side-by-side perspective views of this $\mathrm{HGVC}$ on a $300 \mathrm{~m}$ diameter $30^{\circ}$ superelevated track are shown. The left view illustrates the HGVC stopped for loading with a Cabin Pivot Angle of $-30^{\circ}$. The right view illustrates the same HGVC with a Cabin Pivot Angle of $30^{\circ}$ required for operation at $2 g$ of hypergravity. 


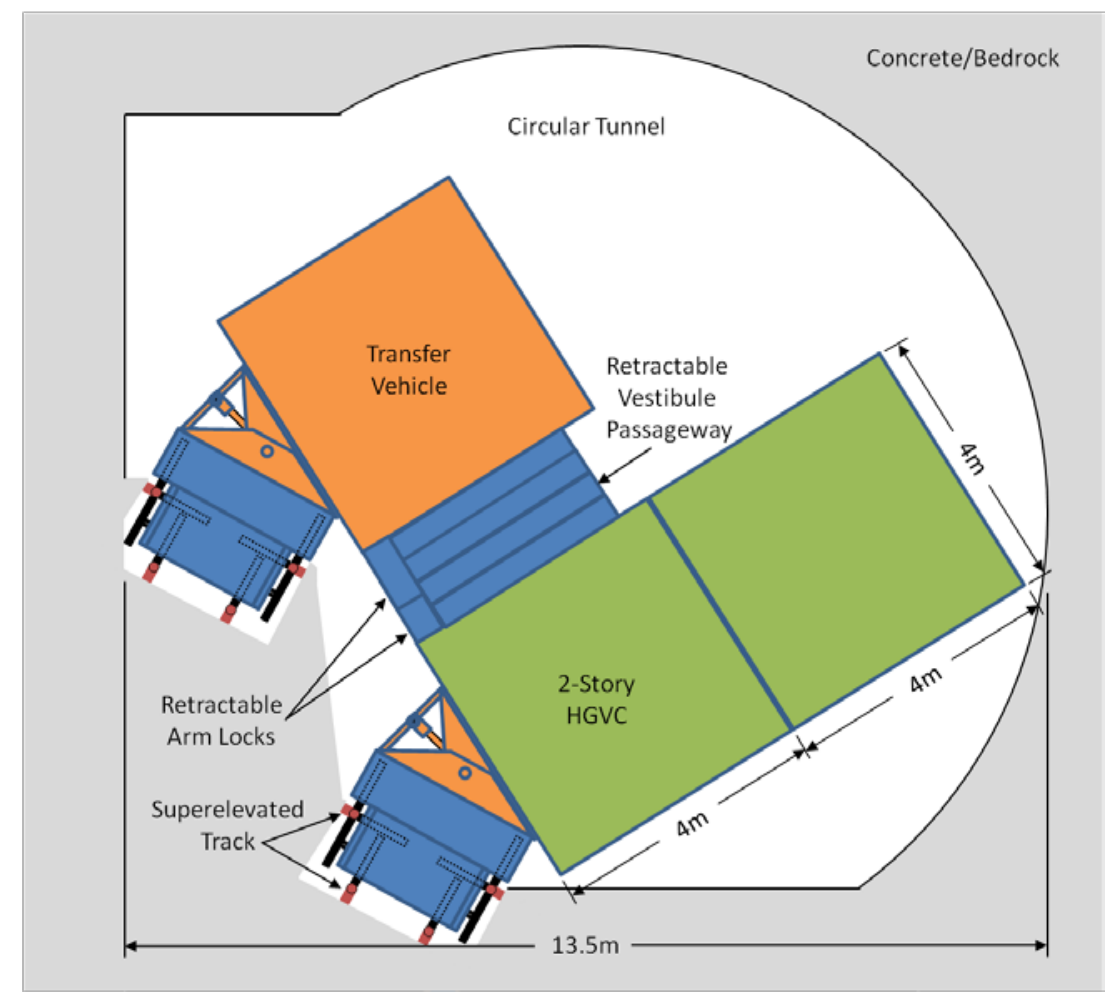

Figure 23. 2-Story HGVC docking with a Transfer Vehicle on a Tunnel Track
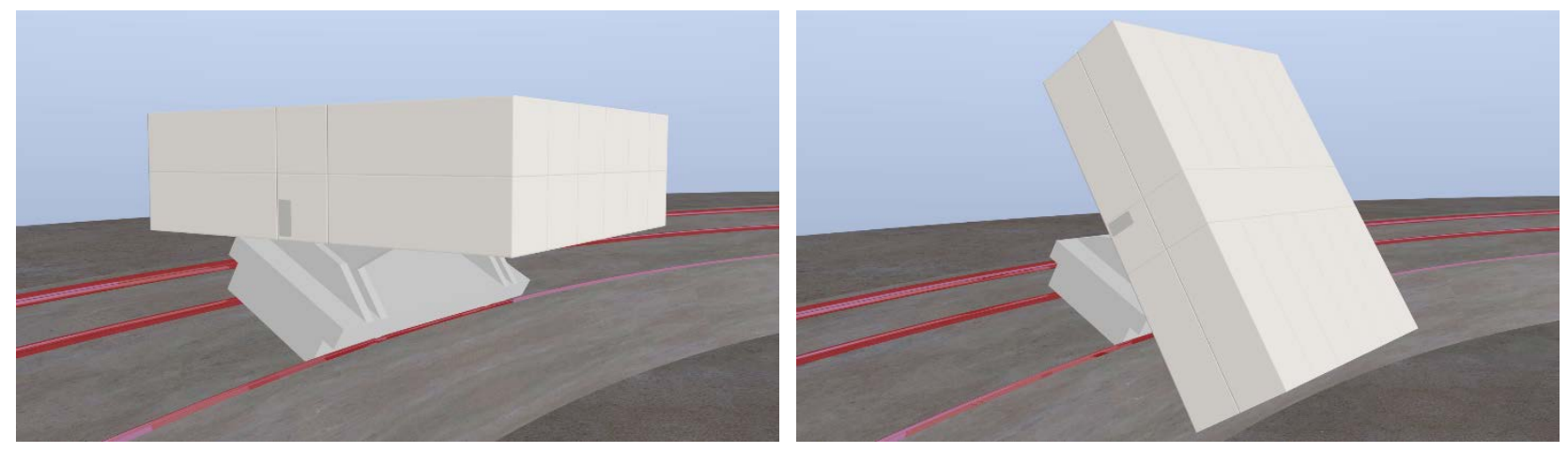

Figure 24. 26-Cabin HGVC on Track Perspective View Stopped (left) and at $2 g$ orientation (right)

This 26-Cabin HGVC is sized to be suitable as an analogue of one of the $3610^{\circ}$ arc rim segments required for a fully-implemented $300 \mathrm{~m}$ dia. wheel-shaped space settlement, such as those depicted in Figs. 1 and 3. This is also a 2-story configuration in which the two center cabins, mounted directly above the HGVC chassis, are $24 \times 4 \times 4 m$ in size. The remaining 24 cabins are half the length $(12 \times 4 \times 4 m)$. Openings and stairways between these cabins can be added as needed. The floor space for this configuration for each HGVC is $1344 m^{2}(14,467$ sq. $\mathrm{ft}$.). 


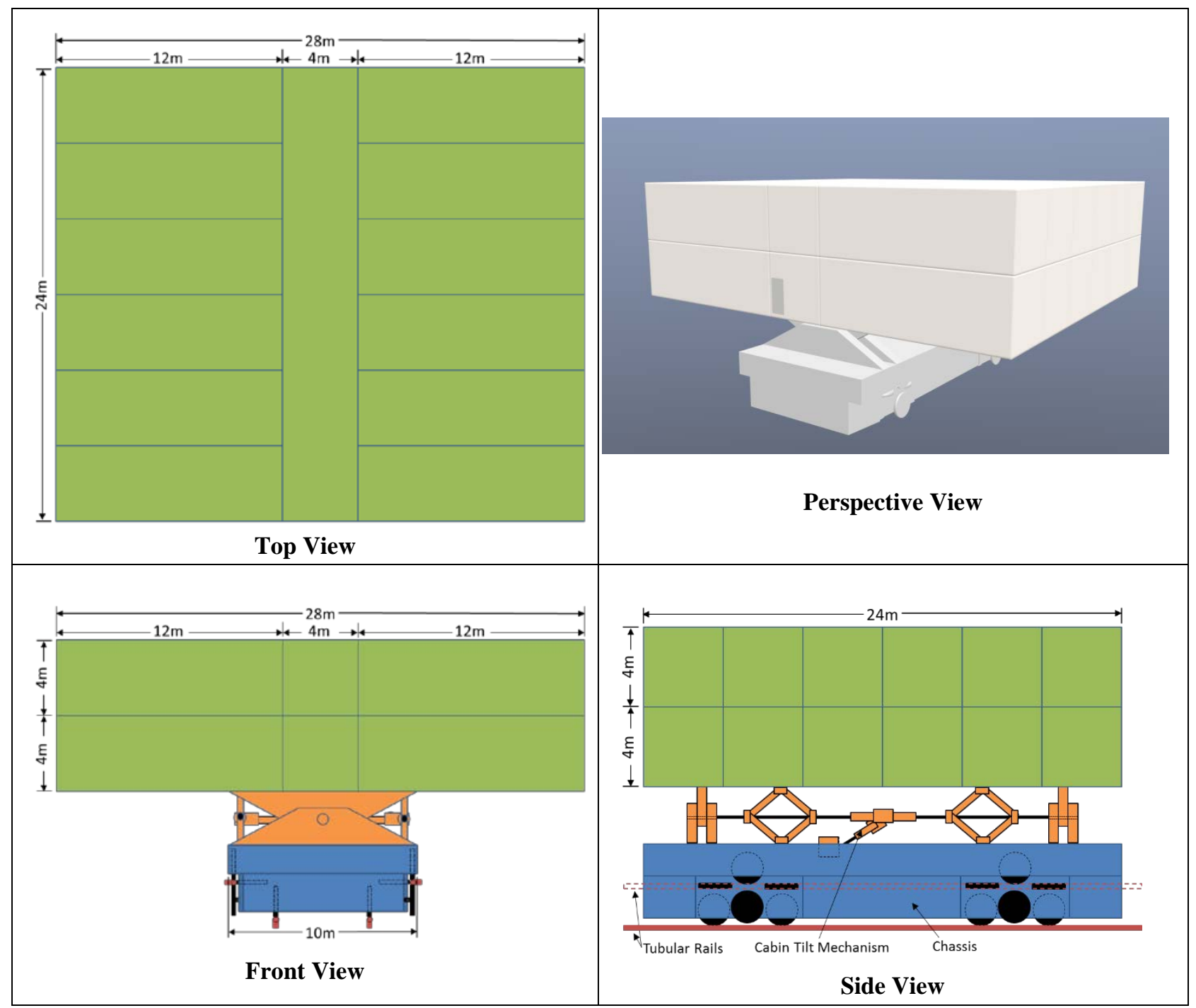

Figure 25. 26-Cabin HGVC Views (2-stories, 13 cabins per story)

The tracks and the HGV chasses can be designed to support the loads as needed. In most cases, the 4-rail track described in this design concept should be sufficient. The HGVC design specifies 10 wheels per wheel truck and 2 wheel trucks per chassis (Fig. 15). To provide context, cargo trains are currently operational that support 40,000kg per 2-wheel axle. ${ }^{6}$

\section{ESHGF Hub-and-Spoke Subsystem Architecture}

An alternative to the Transfer Vehicle is a rotating Hub-and-Spoke subsystem in which the Hub is located at the center of the concentric tracks and rotates at the same velocity as the HGV. This architecture is a closer analogue to that anticipated for an orbiting space settlement. Fig. 26 depicts a single HGV consisting of 36 HGVCs, each with 26 cabins as defined in section II.H.2. To accommodate these HGVCs on Earth, a 315m diameter track is required (see appendix). With each HGVC vertically oriented such that the top faces the track center, as would be the case for an on-orbit implementation, the spacing between the HGVCs would be reduced, reducing the overall diameter to $300 m$.

The total cabin deck area of this configuration is $48,384 m^{2}\left(520,801 \mathrm{ft}^{2}\right)$, excluding the Spoke area and not accounting for deck area lost to accommodate Spoke connections. This deck area supports a population of 1,728 at cruise ship density. Note that this configuration can be implemented incrementally, one cabin or one HGVC at a time. 
As depicted in Fig. 26, each Spoke projects from the Hub and attaches to HGVCs. This figure shows the Spokes connecting to the end of the cabins to simplify the illustration, but designs that entail the removal of some HGVC cabins and connecting each Spoke directly to an HGVC chassis at its midpoint or between two adjacent HGVCs at their vestibule passageway are viable alternatives. Several methods to attach the Spokes to the HGVCs are feasible and require further analysis. The Spoke/HGVC connection design is beyond the scope of this document.

Within each Spoke are passageways to transfer people, animals, plants, and supplies between the Hub and HGVC instead of using a Transfer Vehicle. Each $4 m$-wide Spoke provides ample room for a pair of tilting elevators (or escalators), a cargo cart passageway, and a service passageway as well as conduits for wires, fibers, and pipes to transfer power, data, and fluids respectively. The Spokes may be supported by trolleys running on concentric tracks underneath them and by suspension cables from the Hub peak as needed.

The Hub-and-Spoke subsystem can also support multiple HGVs in concentric rings as shown in Fig. 27. An ESHGF with multiple rings has the advantages of increasing capacity as well as providing an increased variance in hypergravity and rotation radii to better characterize their different effects. For example, an orbiting space settlement may require multiple gravity levels to support crew and visitors preparing for or recovering from low-gravity environment missions.

Fig. 27 depicts three concentric HGVs consisting of a total of 72 HGVCs, each with 26 cabins. The outer HGV is identical to the 36 car HGV shown in Fig. 26. By adding two concentric HGVs, one with 24 HGVCs and the other with 12 HGVCs, the capacity is doubled. The total cabin deck area of this configuration is $96,768 \mathrm{~m}^{2}$ $\left(1,041,601 \mathrm{ft}^{2}\right)$. This supports a population of 3,456 at cruise ship density.

Similar to a rotating restaurant on a tower, the central Hub either can be designed to entirely rotate on a small concentric track of trolleys or be designed as a cylinder rotating around a stationary vertical axle. At rotation rates of 2-3RPM transitioning between the rotating and stationary portions of the Hub would be similar to getting on and off an escalator. The Hub can be connected to the MCC by a tunnel under the ESHGF and/or a bridge.

The Hub-and-Spoke subsystem has the advantage of allowing a continuous flow of people, animals, plants and supplies on and off the ESHGF as needed. Also, it provides a closer analogue to an off-Earth artificialgravity facility in which the Hub would be at the ambient gravity of the ESHGF location, e.g., microgravity on orbit, and Mars gravity on Mars.

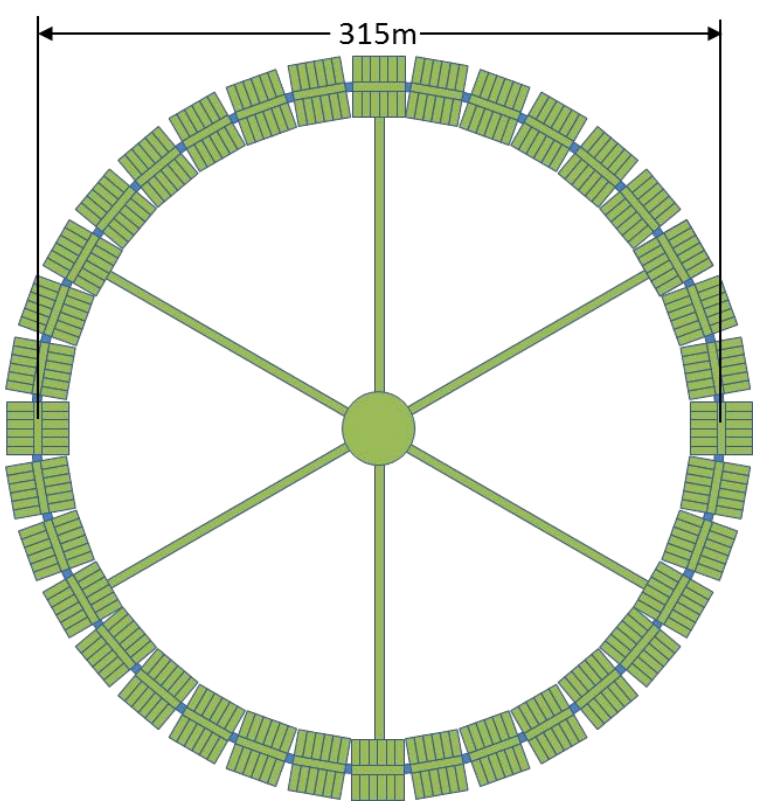

Figure 26. Single Ring 26-Cabin 36-HGVC ESHGF Top View

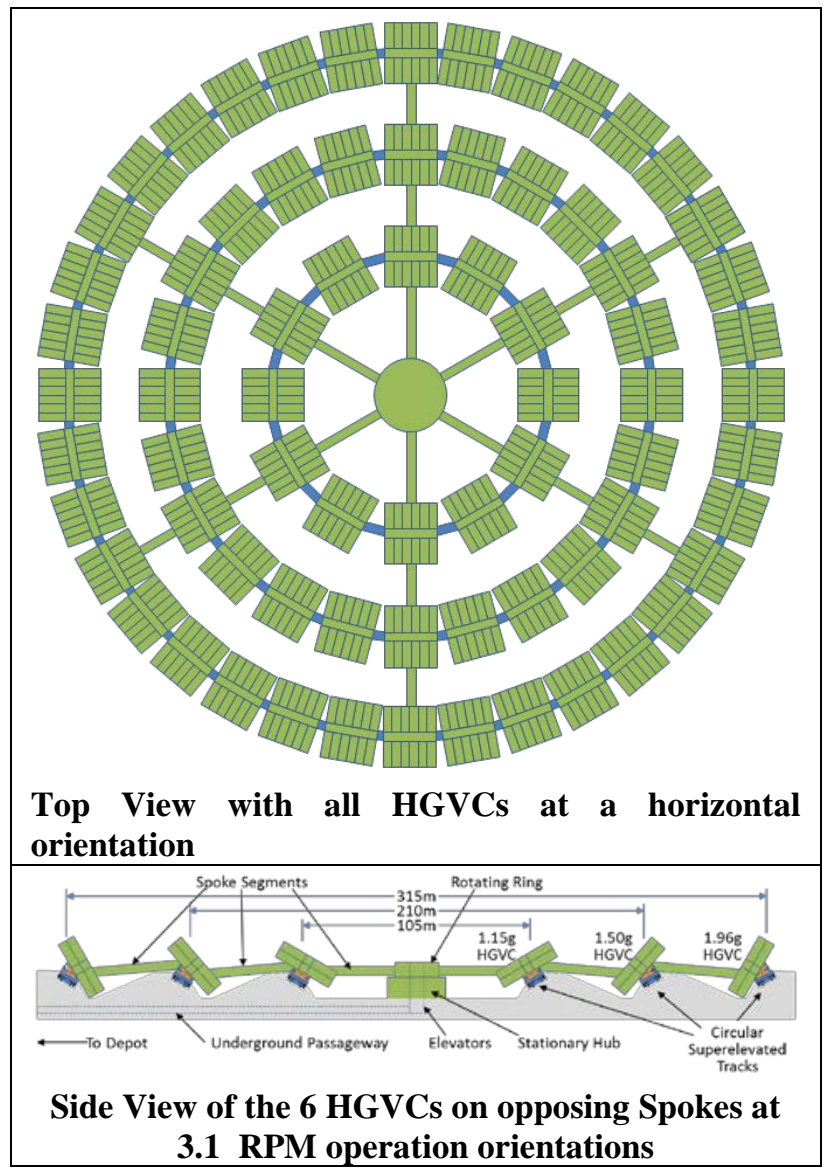

Figure 27. Triple Ring 26-Cabin 72-HGVC ESHGF 
4. ESHGF Hub-and-Spoke Shell

Unlike a torus shell or tunnel that can be used to protect an HGV that uses a Transfer Vehicle, as shown in Fig. 18, a different shell design is required for the optional shell for a Huband-Spoke ESHGF. This shell design can use a stadium roofing material. However, unlike stadiums which do not use a center support, this ESHGF shell uses a stationary $45 \mathrm{~m}$ tower at the center of the ESHGF hub, essentially acting as the axle that the hub ring rotates around, but also is used to support the roof.

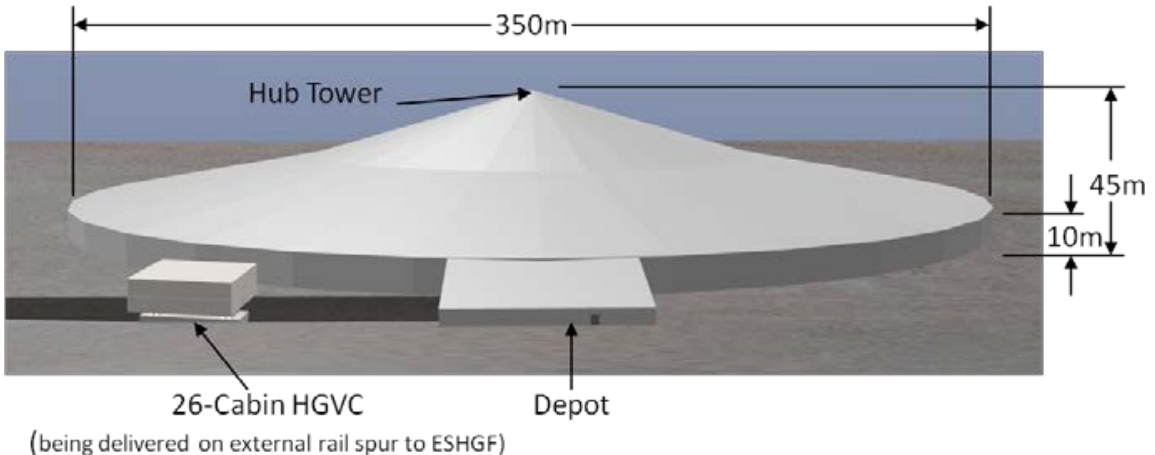

Figure 28. ESHGF Exterior for a Hub-and-Spoke HGV with a $315 m$ dia. Track

Suspension cables are attached from the center tower peak to a circular outer wall that surrounds the ESHGF on the other end. The combination of the center tower, the suspension cables, and the $350 \mathrm{~m}$ dia. $\mathrm{x} 10 \mathrm{~m}$ high outer wall support the ESHGF roofing material as depicted in Fig. 28. This exterior is suitable for housing the Triple Ring 26Cabin 72-HGVC ESHGF shown in Fig. 27. The interior of the ESHGF shell can also be slightly pressurized to provide additional support if required.

For a higher-fidelity ESHGF analogue, the entire facility can be implemented underground to test pressurized HGVCs operating in a vacuum as needed.

\section{ESHGF Hub-and-Spoke Subsystem Incremental Implementation}

The ESHGF Hub-and-Spoke Subsystem can be incrementally implemented in a variety of configurations. An incremental implementation of an ESHGF on Earth can also be used to reflect how an on-orbit artificial-gravity facility may be incrementally implemented to help mitigate its developmental risks. The following figures illustrate how different configurations of 26-Cabin HGVCs can be extended. Similarly, the number of cabins for each HGVs can be incrementally increased starting with single-cabin HGVCs.

The 'I" configuration shown in Fig. 29 , also referred to as the "dumbbell" configuration, is a 2-Spoke configuration that is a relatively low-cost and is a reasonable configuration when lowcapacity is suitable. This configuration has a disadvantage when implemented onorbit that the lengthwise axis is not stabilized when it rotates about the Hub. ${ }^{67}$ However, this can be passively mitigated by adding HGVCs at the outer end of each Spoke as shown in the bottom of Fig. 29.

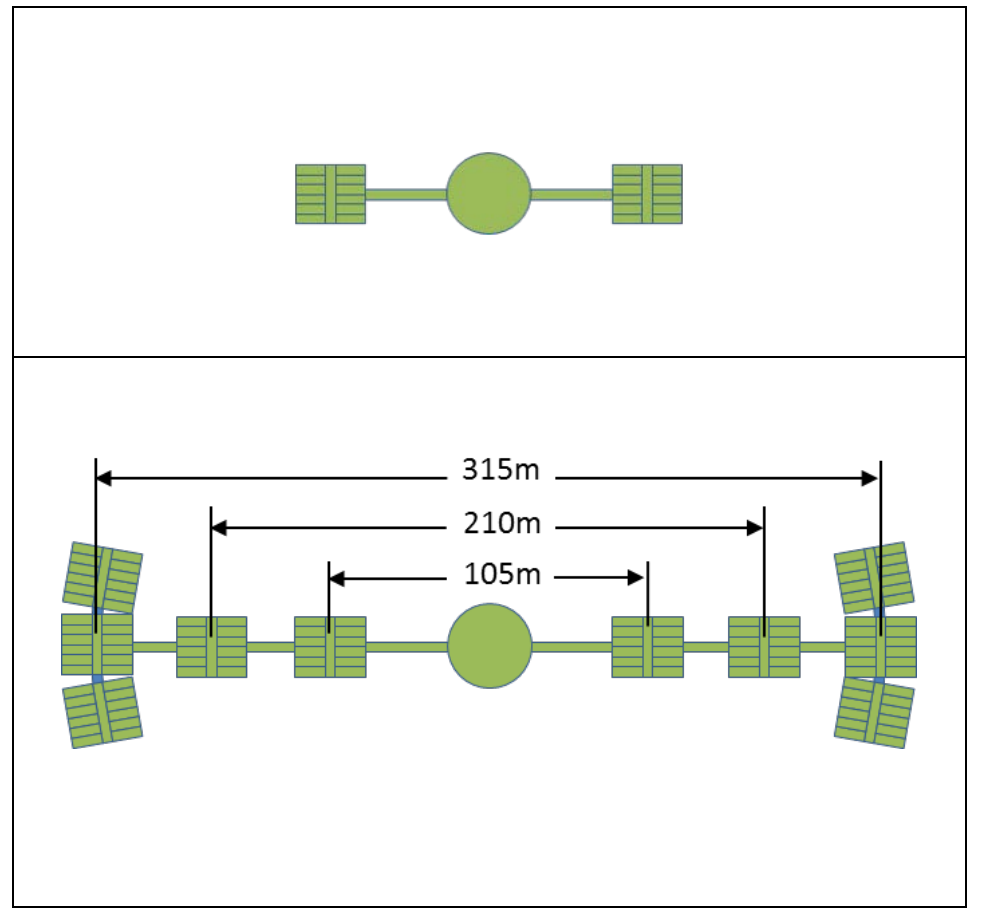

Figure 29. ESHGF Incremental Implementation with 26-Cabin HGVCs in 'I' Configuration Top View Implementations: 2-HGVC (top), 10-HGVC (bottom) 


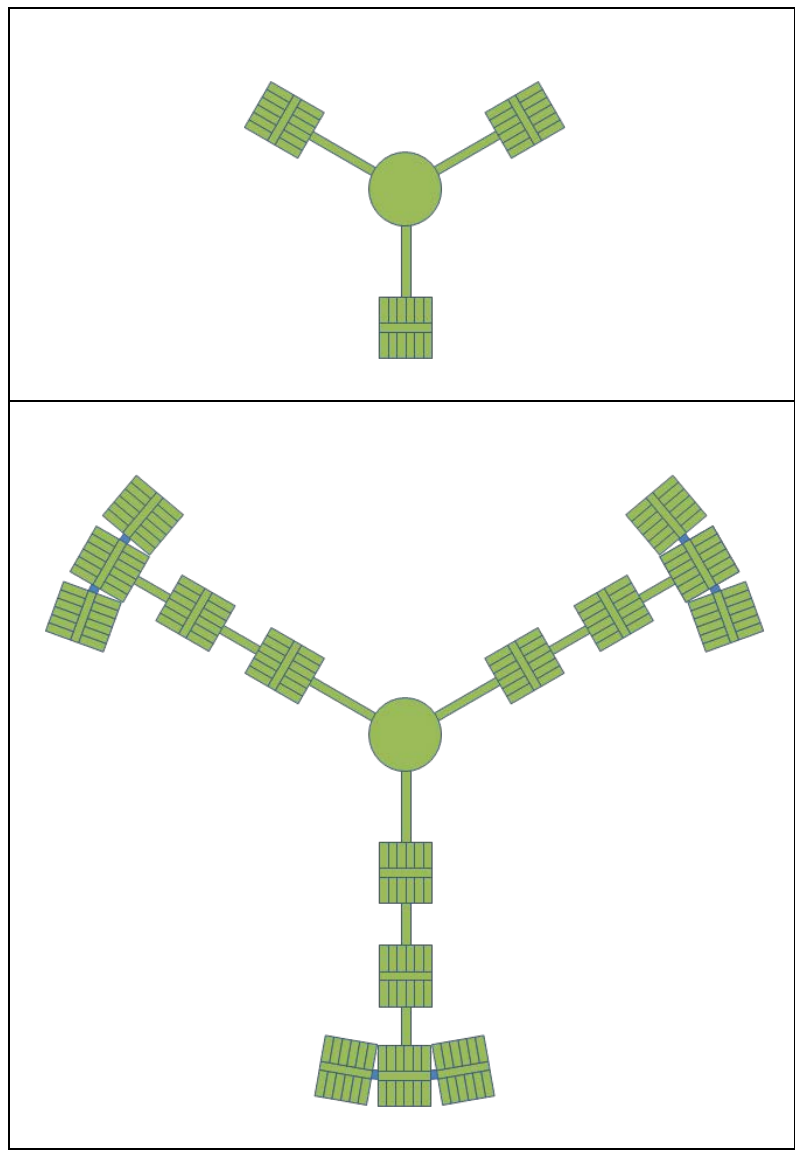

Figure 30. ESHGF Incremental Implementation with 26-Cabin HGVCs in ' $Y$ ' Configuration Top View Implementations: 3-HGVC (top), 15-HGVC (bottom)

The ' $\mathrm{Y}$ ' configuration shown in Fig. 30 is a 3Spoke configuration that has the benefit of being spinstabilized in all three axes when rotated. Also, on-orbit two ' $\mathrm{Y}$ ' configurations can be combined into a single 6-Spoke configuration as shown in Fig. 27 or attached at their hubs and counter-rotated to keep the net angular momentum of the combined facility near zero regardless of the rotation rate. This has the benefit of not requiring propellant to despin if necessary.

The ' $X$ ' configuration shown in Fig. 31 is a 4-Spoke configuration that has the benefit of being spin-stabilized in all three axes when rotated as is the ' $\mathrm{Y}$ ' configuration. It also supports a high-density of HGVCs when increased capacity is desired. An ' $\mathrm{X}$ ' configuration can be created by combining two 'I' configurations when needed. A 6Spoke configuration can be created by combining a 4-Spoke ' $\mathrm{X}$ ' configuration with a 2-Spoke 'I' configuration.

Conversely, once the first on-orbit, modular space settlement is constructed using this architecture, additional settlements can be constructed attached to the same hub that is lengthened to support another "layer" and then separate at the hub once completed. By this time, it may not be necessary to create a ground analogue of a multilayer space-settlement that is replicating, but it is an option. 


\section{Concept of Operations}

The ESHGF can be operated in a number of ways (use concepts) as well as operated in different configurations at different development stages. This section concludes with day-in-the-life examples.

\section{A. Operation Mode Concepts}

The operation mode concepts are summarized in Table 3, each of which is discussed in this section. The operation mode of each HGVC in a HGV can be independently changed during operation to provide needed flexibility.

Table 3. Operation Mode Concept Summary

\begin{tabular}{|c|c|c|c|}
\hline $\begin{array}{l}\text { Operation } \\
\text { Mode } \\
\text { Description }\end{array}$ & Rationale & $\begin{array}{l}\text { HGV } \\
\text { Operation } \\
\text { Duration }\end{array}$ & $\begin{array}{l}\text { Human } \\
\text { Occupant } \\
\text { Duration }\end{array}$ \\
\hline $\begin{array}{l}\text { Unoccupied } \\
\text { short-term } \\
\text { operation }\end{array}$ & $\begin{array}{l}\text { - Perform short-term HGV testing } \\
\text { - Short-term plant and animal experiments }\end{array}$ & $<1$ day & 0 \\
\hline $\begin{array}{l}\text { Unoccupied } \\
\text { continuous } \\
\text { operation }\end{array}$ & $\begin{array}{l}\text { - Long-term HGV testing } \\
\text { - Long-term plant and animal experiments }\end{array}$ & Indefinite & 0 \\
\hline $\begin{array}{l}\text { Occupied } \\
\text { short-term } \\
\text { operation }\end{array}$ & $\begin{array}{l}\text { - Evaluate short-term hypergravity effects on humans } \\
\text { - Perform tasks requiring humans } \\
\text { - Support short-term visitors } \\
\text { - Transfer Vehicle not required } \\
\end{array}$ & $<1$ day & $<1$ day \\
\hline $\begin{array}{l}\text { Continuous } \\
\text { operation with } \\
\text { short tours of } \\
\text { duty }\end{array}$ & $\begin{array}{l}\text { - Long-term HGV testing } \\
\text { - Plant and animal experiments } \\
\text { - Evaluate short-term hypergravity effects on humans } \\
\text { - Perform short-term treatments on humans } \\
\text { - Perform tasks requiring humans } \\
\text { - Support short-term visitors } \\
\end{array}$ & Indefinite & $<1$ day \\
\hline $\begin{array}{l}\text { Continuous } \\
\text { operation with } \\
\text { long tours of } \\
\text { duty }\end{array}$ & $\begin{array}{l}\text { - Long-term HGV testing } \\
\text { - Plant and animal experiments } \\
\text { - Evaluate long-term hypergravity effects on humans } \\
\text { - Perform long-term treatments on humans } \\
\text { - Perform tasks requiring humans } \\
\text { - Support short-term visitors } \\
\text { - Artificial-Gravity Space Settlement Analogue }\end{array}$ & Indefinite & Indefinite \\
\hline
\end{tabular}

\section{Unoccupied short-term operation mode}

The Unoccupied short-term operation mode is expected to be used for initial testing of the HGV in the ESHGF. The HGV may also be tested in this mode on a commercial track.

In the ESHGF, the HGV can be used to characterize the short-term effects of the HGV on objects, animals, and plants prior to a HGV being used by humans.

\section{Unoccupied long-term operation mode}

The HGV, or a subset of its HGVCs, can be used to characterize the long-term effects of hypergravity and lowRPM high-radius rotation on objects, manufacturing processes, animals, and plants without requiring the presence of humans, e.g., perform science regarding the multi-generational effects of hypergravity on plants, fish, birds, mammals, etc....

\section{Occupied short-term operation mode}

Once the HGV has been tested without humans and certified as safe for human occupancy for short-terms, the HGV can be used to evaluate short-term hypergravity effects on humans.

The HGV can also be used for demonstrating the system capabilities for short-term visitors. High-density population HGVCs may be dedicated for this purpose. 
When the entire HGV is operated in this mode, the Transfer Vehicle is not required since the entire HGV can stop at the DCC for loading and unloading.

4. Continuous operation with short tours of duty mode

A primary purpose of this mode is to study the hypergravity/rotation effect on people to determine the extent that the tours of duty can be safely extended to indefinite durations.

Assuming that by performing short-term tests on people it is determined that there is a therapeutic benefit for people to undergo hypergravity for short periods, the HGV could be used in this mode to perform such treatments, e.g., strength building, osteoporosis mitigation, and extended microgravity rehabilitation.

This mode also can be used for HGVCs being used for the unoccupied, long-term operation described in section III.A.2, but tasks requiring humans for short-terms are needed, e.g., perform animal examinations and maintenance that can be performed during operation.

Another purpose for this mode is to study the periodic effects of hypergravity on humans. For example, an experiment may characterize any differences in the effects of hypergravity between two groups of people; one group who for 180 days alternates between being onboard the HGV for 7 days then off for 7 days compared with the other group which alternates between being onboard for 12 hours then off for 12 hours each day for the same 180-day period.

\section{Continuous operation with long tours of duty mode}

A primary purpose of this mode, supporting long tours of duty, is to characterize the long-term hypergravity/rotation effects on people, animals, and plants to determine if they can safely live indefinitely in longradii rotating artificial-gravity facilities as well as to determine if there are long-term benefits to living in a hypergravity environment. If such benefits do exist, then these facilities can be used for therapy.

Also, an HGV in this mode can be used as an analogue to study and experience what life might be like in longradii rotating artificial-gravity settlements orbiting Earth, on the Moon, on Earth-Mars cycler orbits, ${ }^{68}$ and on Mars and its moons.

\section{B. ESHGF Configurations}

The extensible, modular design of the ESHGF supports it being operated in a wide variety of configurations in which its capabilities can be incrementally implemented over time. The following configurations are listed in the proposed order of development.

\section{Single-Car Single-Cabin HGV Initial Checkout on Commercial Track}

The simplest configuration is a single HGVC with a single cabin that runs on a commercial track. The basic concepts and systems of the HGVC can be tested prior to building the ESHGF track.

\section{Single-Car Single-Cabin HGV on ESHGF Track}

The next configuration is to run the single-cabin HGVC on the superelevated circular ESHGF track.

\section{Multi-Car Single-Cabin HGV}

This configuration includes all HGVs with multiple single-cabin HGVCs. In the maximum implementation of this configuration, the HGVCs fill the entire circular track and are connected such that the first HGVC in the front of the HGV is connected to its rear HGVC forming a ring of HGVCs.

\section{Multi-Car Single-Cabin HGV with Transfer Vehicle}

As the capacity of the HGV increases, implementing a Transfer Vehicle and track in the ESHGF may be warranted. As discussed in section II.C, a Transfer Vehicle can be used to efficiently transfer people and cargo on and off the HGV without stopping the HGV. Doing so is less disruptive for HGV operations and less costly by reducing the energy and maintenance costs with stopping and starting the entire HGV.

\section{Single-Car Multi-Cabin HGV}

Another configuration option to increase capacity is to add modular cabins to a HGVC. A 26-cabin HGVC is discussed and depicted in figures in section II.H.2. However, a wide variety of multi-cabin HGVC configurations are feasible and can be implemented so that they can be reconfigured and/or upgraded on demand. 
Multi-cabin HGVC configurations facilitate larger habitable spaces, shorter point-to-point distances compared to multiple single cabin HGVCs with the same volume, and provide more variation in rotation radii and hypergravity due to cabin points being further from the track gauge center.

A multi-cabin HGVC can also be configured to more closely replicate a potential rim section of an orbiting settlement when being used as a space settlement analogue.

\section{Multi-Car Multi-Cabin HGV}

Just as multiple single-cabin HGVCs can be configured as discussed in section III.B.3, capacity can be increased with multiple multi-cabin HGVCs. It also facilitates both HGVC specialization and the simultaneous operation of different multi-cabin HGVC configurations for comparison.

\section{Multi-Car Multi-Cabin HGV with Transfer Vehicle}

The purpose and configuration of a Multi-Cabin HGVC are similar to those of a single-cabin HGVC and Transfer Vehicle as discussed in section III.B.4, except that the capacity of each HGVC is increased. An example of a 2-Cabin HGVC with a Transfer Vehicle is shown in Fig. 23.

\section{Multi-Car Multi-Cabin HGV with Hub \& Spokes}

Assuming that the benefits of an ESHGF are confirmed using a single-car HGV, a multi-car multi-cabin HGV with a central Hub \& Spokes configuration, as described in section II.H.2, is recommended to increase HGV capacity and its flexibility to continuously transfer people on and off the HGV, as well as to obtain a closer analogue to an orbiting space settlement,. This configuration can be incrementally implemented by operating the ESHGF in some of the configurations described above first.

\section{ESHGF Use Case Examples}

This section describes the operation of an ESHGF with three use case examples.

\section{Short-Term Occupied Mode with Single-Cabin Single-Car HGV}

The HGV is sitting on a $30^{\circ}$ superelevated track parked next to the DCC. The single HGVC cabin is pivoted at a $-30^{\circ}$ so that the cabin floor is level. A retractable bridge (similar to an airport jetway) is extended from the DCC to the HGV. The cargo is loaded followed by the loading of the crew and passengers who proceed to their assigned rooms. Once loaded, the HGV door is sealed and the DCC bridge is retracted.

A brief announcement over the intercom informs the passengers that the HGV is embarking and that they will reach the operating hypergravity level of $2 g$ 's in 10 minutes. Unlike an airplane, at all times the people are free to continue to move about the HGV. The HGV slowly accelerates at a rate that is barely perceptible to the passengers and the HGVC cabin pivot angle also slowly increases and is not perceptible. The passengers eating in the cafe continue dining without taking notice. The beverage glasses resting on the tables give no indication that the entire cabin is tilting as the HGV accelerates. After 10 minutes, the HGV cabin hypergravity is 2g's and the cabin pivot angle is now $30^{\circ}$. Combined with the $30^{\circ}$ track back angle, the cabin floor angle is $60^{\circ}$ with respect to the ground, but it seems level to all onboard. Just after the HGV reaches its operation speed, a passenger in the cafe notes that his glass does feel heavier since it now weighs $100 \%$ more as well as his arm. Another passenger notes that she feels like she is wearing armor when she stands up as the passengers begin to adjust to the hypergravity they are experiencing.

After a full day of operation, another announcement over the intercom informs the passengers that the HGV will be stopping in 10 minutes and to prepare to disembark. The HGV slowly begins to decelerate at an imperceptible rate, e.g., $0.07 \mathrm{~m} / \mathrm{s}^{2}$. Simultaneously, the cabin also begins to pivot back to $-30^{\circ}$ also at an imperceptible rate of $0.1^{\circ}$ per second such that the cabin stops pivoting at the same moment the HGV stops at the DCC. The DCC bridge is extended to the HGV and the passengers and their cargo then disembark as the HGV is prepared for its next tour. As the passengers leave, they feel like they are practically floating as their bodies begin to readjust to the lower gravity of Earth.

\section{Multi-Cabin Multi-Car Nominal HGV Operations with a Transfer Vehicle}

The HGV is configured and operates like a small cruise ship with office and manufacturing areas to continuously support 200 crew and passengers who live and work there continuously for months along with a stream of daily visitors. In addition, the HGV is configured with life science areas devoted to raising and studying generations of plants and animals subject to hypergravity and rotation. Medical areas are also devoted to ensuring the safety of all onboard as well as studying the hypergravity and rotation effects on the people. All the HGVCs are two cabins high 
providing two decks. There are several large openings between these two decks to provide a more spacious atmosphere as well as support the option for rooms with balconies on the second deck and space for trees to grow from the first deck into the second deck cabins.

The Transfer Vehicle is scheduled for over 10 trips per day to load and unload people and cargo on the HGV while the HGV speed remains constant. For each trip, the Transfer Vehicle stops at the DCC; people and cargo unload followed by the loading of people and cargo on their way to the HGV. A water tank located in the Transfer Vehicle chassis is also loaded and a sewage tank is unloaded as needed. The Transfer Vehicle disembarks and within 10 minutes it reaches the operational velocity of the HGV and prepares to dock by matching the pivot angle of its cabin to the pivot angle of the HGVC it is docking with. As the Transfer Vehicle extends its forward retractable arm lock and slowly approaches the target HGVC from the rear, the HGVC extends its forward retractable arm lock to lock with the forward retractable arm lock of the approaching Transfer Vehicle. Once the two front arms make contact and lock, the rear retractable arm locks on both vehicles are extended and locked. These locks are designed to secure both cabins to each other while docking, but for safety reasons either vehicle can unilaterally disengage these locks in an emergency. Once both pairs of retractable arm locks are locked, a retractable vestibule is extended from the Transfer Vehicle to an HGVC cabin and then the transfer of people and cargo between the two vehicles begins. In addition, underneath this vestibule pipes are connected and the transfer of water and sewage commences. This docked configuration of a Transfer Vehicle with a single-cabin HGVC is illustrated in Figs. 16, 17, and 20.

After 10 minutes, the vehicles prepare to undock. The fluid transfer pipes are disconnected and the retractable vestibule is retracted followed by the unlocking and retraction of the two pairs of retractable arm locks. Once undocked, the Transfer Vehicle decelerates over the next 5 minutes and stops at the DCC where it repeats this process.

\section{Multi-Ring, Multi-Cabin, Multi-Car Nominal HGV Operations with a Hub and Spokes}

The ESHGF is configured as shown in Fig. 27 and operates as a small city with its own government, a permanent space-settlement-like environment for 500 permanent residents and 1000 visitors/commuters. The HGVCs and their cabins continue to be produced by a wide variety organizations according to a publically-available interface specification. Some of these HGVCs and cabins are delivered to the ESHGF to be swapped in during maintenance periods while the others are used as stationary space-settlement analogues at academic and research institutions. Some of the cabins produced are engineering units of cabins designed for eventual space operation.

Three HGV rings generate three different gravity levels, $1.15 g, 1.5 g, 2 g$, for research, training options, as well as to reduce the effects of sudden gravity changes. Several of the HGVCs are used as biospheres to create a wide variety of habitats from dessert to tropical. In addition to the housing for the residents, the facility has several hotels to cater to the overnight visitors and conference attendees. The facility also features several medical clinics that specialize in treating a variety of conditions with hypergravity, such as osteoporosis and obesity. Several of the HGVCs are used by the NASA astronauts, their support teams, and families. Prior to a trip to space and immediately after returning, astronauts live and train in this facility to mitigate the effects of microgravity in space. The facility also leases HGVCs to several sport teams and fitness organizations for training as well. Manufacturing focuses on the in-house development of replacement components, including cabin module construction, from raw ore and nonorganic waste. Similarly, farming focuses on food production and approaching $100 \%$ recycling in an effort to become self-sufficient and eliminate nearly all organic waste, which is desirable for an efficient space-settlement, particularly beyond LEO.

People enter and exit the HGVs through the Hub and Spokes (there is no transfer vehicle). The Hub core is stationary and contains an elevator from the spoke deck level to its base at the end of an underground passage for trams that transfer people and cargo between the Hub base and outside the facility. The outer portion of the Hub rotates at 3.1 RPM, the same rate as the HGVs and Spokes. People move from the stationary center to the rotating outer band of the Hub deck like stepping on an airport moving walkway, except that it is circular. From the Hub deck rotating band, people choose one of the elevators or escalators in the six Spokes to go to the destination of their choice, returning to the Hub deck the same way.

\section{Conclusion}

The design concept for an extensible, modular, Extended-Stay HyperGravity Facility (ESHGF) was presented. The primary purpose of the ESHGF is to produce a flexible suite of precursor facilities suitable for researching the feasibility of extending the permanent presence of life beyond Earth in artificial-gravity settlements. but an ESHGF is expected to provide a variety of other benefits as well. The ESHGF produces an adjustable hypergravity in a $300 \mathrm{~m}$ diameter rotating environment for people to work and live in along with animals and plants for long-term periods. 
Two key features of this environment are that the interior of the rotating facility is effectively both motionless and level with respect to its occupants and cargo while the hypergravity is generated.

The design consists of modules that can be assembled and disassembled as needed to be transported, to change system capacity, to change module purposes, and to upgrade/maintain modules while keeping the overall system operational. The modules are sized for transport, including transport by heavy-lift launch vehicles for operation offEarth.

For this design concept, an incremental implementation approach is recommended starting with a single-car hypergravity vehicle with a $24 \times 4 \times 4 m$ cabin on a 300m diameter $30^{\circ}$ superelevated track as depicted in Fig. 11 capable of a hypergravity range of $1-2 g$. Additional cars and cabins can be added as needed to increase the capacity and fidelity of the ground-analogue up to a hub-and-spoke space settlement as shown in Figs. 26 and 27.

Micro-gravity is known to negatively affect human health and limits the duration people can live off Earth and return. This facility will permit research on the long-term health and behavioral effects of various artificial-gravity levels and rotation rates on humans and other life, among other things, to establish the design requirements for longterm space settlements. In order to enable long-term human missions off-Earth, the National Academies Institute of Medicine committee recommended for NASA to focus on giving, "increased priority to understanding, mitigating, and communicating to the public the health risks of long-duration missions beyond Earth orbit" and "using more extensively analog environments that already exist and that have yet to be developed;". ${ }^{4}$ Implementing artificialgravity settlements and understanding their effects on life appear to be crucial for permanently extending life beyond Earth. The International Academy of Astronautics Study Group on artificial gravity recommended that, "the most efficient means of developing an effective flight artificial gravity countermeasure is by appropriate and timely use of ground facilities". ${ }^{5}$ An NRC report on the NASA Space Technology Roadmaps states, "The panel identified Artificial Gravity Evaluation/ Implementation as a game-changing capability that would greatly mitigate many adverse health effects that would otherwise occur during long-duration habitation in transit (or Earth orbit)." ${ }^{6}$ In order to address these challenges, an ESHGF implemented on Earth is a rational next step for extending the permanent presence of life beyond Earth.

\section{Appendix}

\section{A. HGVC Metrics}

As defined in Eq. (1), hypergravity is a function of the distance of each cabin point relative to the track circle center. One of the parameters required is the distance from a HGVC cabin point to the center of the track circle. This distance, $r_{p o s}(x, y, z, P)$, i.e., the radius for a given cabin floor point $\left(x, y, C_{F T}\right)$ coordinate at Cabin Pivot angle $P$, is defined by Eq. (4). The HGVC Cabin coordinate frame is defined in section II.F.1 and depicted in Fig. 22.

$$
r_{\text {pos }}(x, y, z, P)=\sqrt{x^{2}+\left(r_{T}+r_{B}+r_{C O}+r_{P}(y, z, P)\right)^{2}}
$$

Superelevating the track by angle $T_{B}$ has the effect of shifting the cabin pivot-axle centerline toward the track center by $r_{B}$ per Eq. (5).

$$
r_{B}=-\sin \left(T_{B} \cdot \pi / 180\right) \cdot P_{h}
$$

Similarly, pivoting a cabin by angle $P$, in addition to the track superelevation angle $T_{B}$, also has the effect of shifting cabin point radii. The radii of the cabin points in the vertical plane where $x=0$ are offset by $r_{P}(y, z, P)$ per Eq. (6).

$$
r_{P}(y, z, P)=-\cos \left(\cos ^{-1}\left(\frac{-y}{\sqrt{y^{2}+\left(C_{P O}+z\right)^{2}}}\right)-\left(P+T_{B}\right) \cdot \pi / 180\right) \cdot \sqrt{y^{2}+\left(C_{P O}+z\right)^{2}}
$$

Note that when the Cabin Pivot angle offsets the Track Superelevation angle such that the cabin is level, the Cabin Pivot Angle Radius Offset is $y$, i.e., $r_{P}\left(y, z,-T_{B}\right)=y$.

Since the track is curved but the cabin is straight, the center of the cabin, $(0,0,0)$, is off the track circle by an additional offset of $r_{C O}$ per Eq. (7).

$$
r_{C O}=\sqrt{r_{T}^{2}-\left(\frac{C_{L}}{2}-W_{T O}\right)^{2}}-r_{T}
$$


As per Eq. (4), the radius $r_{p o s}(x, y, z, P)$, from the track circle center to a point at coordinates $(x, y)$ on a cabin floor, is the track circle radius $r_{T}$ offset by the track superelevation angle offset, the cabin pivot angle offset, the chassis centerline center offset, and the cabin floor position $(x, y)$ offset.

The track superelevation angle radius offset, Eq. (5), is zero when the superelevation angle is zero, but is equal to the Pivot axle center height, when the superelevation angle $=90^{\circ}$ as would be the case in a micro gravity environment (see Fig. 32).

Similarly, the pivot angle radius offset, Eq. (6), is zero when the pivot angle is equal to the negative of the track superelevation angle (when the HGV is stopped, see Fig. 21). In the case when the sum of the track superelevation angle and the cabin pivot angle is $90^{\circ}$, i.e., when the cabin floor is perpendicular to the track circle plane,

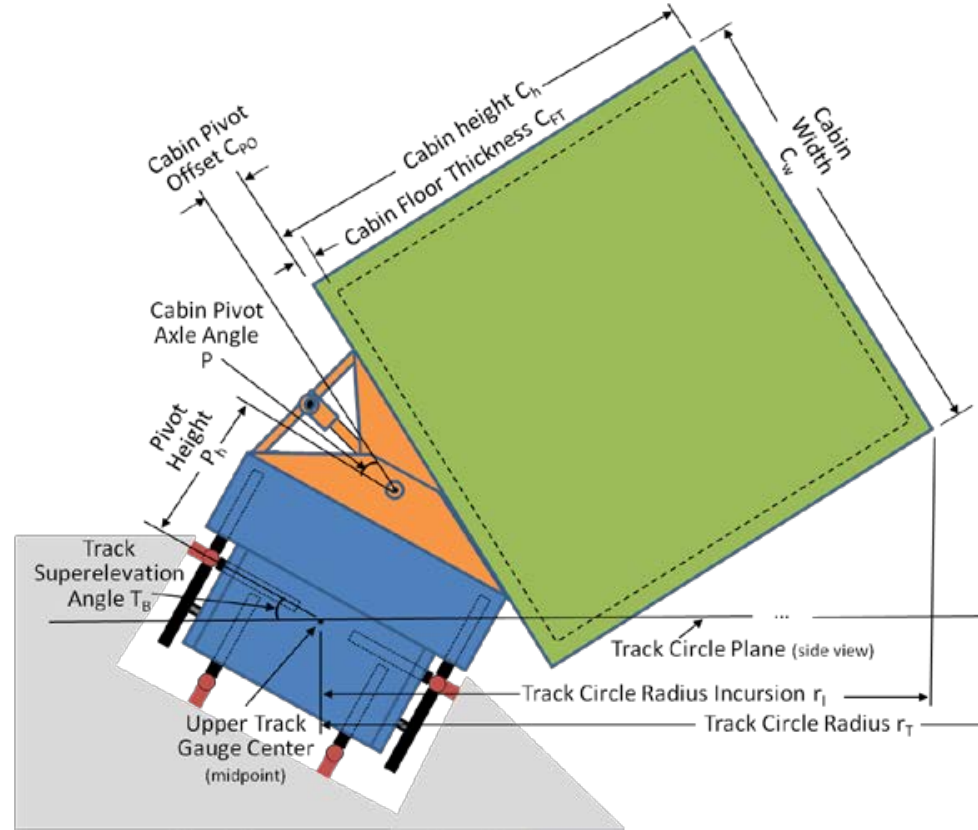

Figure 32. HGVC Tilted Cabin on Track - Rear View the pivot angle radius offset is equal to the cabin floor height from the pivot axle, see Fig. 32. For a "second-story cabin," this height is increased by the height of the first-story cabin. The hypergravity in the second story cabin decreases accordingly.

The chassis centerline center offset is due to the chassis and cabin being straight lengthwise and not being arced at same radius as the track. Consequently, the chassis lengthwise centerline forms a chord intersecting the track circle as illustrated in Fig. 15, where the each truck center-point is the track radius from the track circle center. The chassis centerline center offset is essentially the chord height measured midway between the chassis truck centers.

\section{B. HGVC Track Capacity}

Per Eq. (9), the maximum number of HGVCs that can fit on a circular track, $n_{m x}$, is a function of the track radius, the maximum track-circle incursion distance, the HGVC upper-inner lengthwise cabin edge length at this distance, and the minimum cabin spacing margin, $C_{s m}$, between HGVCs (to avoid collisions between the top inner rear corner of a cabin on an HGVC and the top inner front corner of a cabin on the immediately following HGVC as shown in Fig. 16) when pivoting the cabins. The problem is essentially to maximize $\mathrm{n}$ for an $\mathrm{n}$-gon:

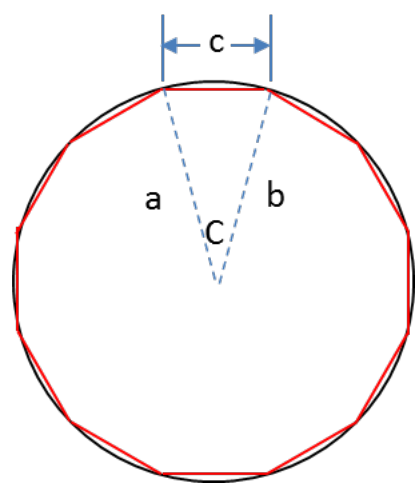

Figure 33. Maximum Dodecagon in a Circle

Fig. 33 depicts a Dodecagon (12-sided n-gon) with its vertices on a circle. The length of each side $c$, with both vertices on the circle, can be calculated using the law of cosines, $c^{2}=a^{2+} b^{2}-2 a b \cos (C)$. This equation is restated in Eq. (8) letting $n=$ number of $n$-gon sides and $r=a=b$.

$$
c=\mathrm{r} \sqrt{2-2 \cos \left(\frac{2 \pi}{n}\right)}
$$

By substituting $C_{T L}+C_{L M}$ for c, and $\mathrm{r}_{\mathrm{T}}-\mathrm{r}_{\mathrm{I}}$ for $\mathrm{r}$, and maximizing n, Eq. (8) is reformulated as Eq. (9).

$$
n_{m x}=\operatorname{trunc}\left(\frac{2 \pi}{\cos ^{-1}\left(1-\frac{\left(C_{T L}+C_{L m}\right)^{2}}{2\left(r_{T}-r_{I}\right)^{2}}\right)}\right)
$$

The maximum radius corner incursion offset of an HGVC cabin, $r_{I}$, occurs 
when the cabin is tilted such that the height of the upper inner cabin edge are the same height as the Cabin Pivot Axle center, which occurs at the Cabin Pivot Angle $P_{\operatorname{Imx}}$ and is defined by Eq. (10).

$$
P_{I m x}=\tan ^{-1}\left(\frac{2\left(C_{T H}+C_{P O}\right)}{C_{T W}}\right) \cdot \frac{180}{\pi}-T_{B}
$$

The maximum radius corner incursion offset, $r_{I}$, is defined by a special case of the Cabin Point Radius equation Eq. (4) where the point is a Cabin roof inner corner (assuming the Cabin is centered on the Chassis) and the Cabin Pivot angle $P=P_{I m x}$, and is parameterized in Eq. (11).

$$
r_{I}=r_{T}-r_{p o s}\left(C_{T L} / 2, C_{T W} / 2, C_{T H}+C_{P O}, P_{I m x}\right)
$$

Once $n_{m x}$ is determined, the actual minimum cabin distance (spacing margin) between top inner cabin corners of adjacent HGVCs , $C_{s m}$, can be determined by Eq. (12).

$$
C_{s m}=\left(\left(\mathrm{r}_{\mathrm{T}}-r_{I}\right) \sqrt{2-2 \cos \left(\frac{2 \pi}{n_{m x}}\right)}-C_{T L}\right) \cdot \sin \left(\frac{\left(n_{m x}-2\right) \pi}{2 n_{m x}}\right)
$$

Consider the following example. For the 26-cabin HGVC shown in Fig. 25 with each cabin $4 m$ high, $4 m$ wide, and the side cabins $12 \mathrm{~m}$ long, $C_{T H}=2 \cdot 4=8 \mathrm{~m}$, and $C_{T W}=4+2 \cdot 12=28 \mathrm{~m}$. For a $300 \mathrm{~m}$ diameter track with a superelevation angle, $T_{B}=30^{\circ}$, a Pivot Axle height of $P_{h}=3.4 \mathrm{~m}$, and a Cabin Pivot Offset $C_{P O}=1.5 \mathrm{~m}$, the Pivot Angle that maximizes the cabin incursion into the track circle is $P_{\operatorname{Imx}}=4.16^{\circ}$ per Eq. (1) and the maximum radius corner incursion offset is $r_{I}=18.3 m$ per Eq. (11), but for a $4 \times 4 \times 24 m$ single-cabin HGVC, $P_{\operatorname{Imx}}=36^{\circ}$ and the incursion offset $r_{I}=5.2 \mathrm{~m}$. Consequently per Eq. (9), with a minimum Cabin length margin $C_{L m}=0.25 \mathrm{~m}$, the maximum number of 26-cabin HGVCs that will fit on the track is $n_{m x}=34$, with a Cabin spacing minimum of $C_{s m}=29 \mathrm{~cm}$ per Eq. (12). Similarly, the maximum number of the single-cabin HGVCs on a $300 m$ dia. track is $n_{m x}=37$ with a Cabin spacing minimum of $C_{s m}=56 \mathrm{~cm}$.

In the above example, only 34 of the specified 26-cabin HGVCs will fit on a $300 \mathrm{~m}$ dia. track. If the system is designed such that $P_{I m x}+T_{B}=90^{\circ}$, i.e., the HGVCs cabins are always perpendicular to the rotation plane as would be the case on-orbit and on low-gravity bodies like the Moon, 36 HGVCs would fit in a $300 m$ diameter circle. On Earth, 36 of the same size HGVCs require a $315 \mathrm{~m}$ track diameter, i.e., $r_{T}=157.5 \mathrm{~m}$, per Eq. (9), due to the maximum cabin incursion offset of $18.36 \mathrm{~m}$ per Eq. (11). This 36-HGVC configuration is described in section II.H.3.

\section{Acknowledgments}

This work was supported by the NASA Space Technology Mission Directorate Small Spacecraft Technology Program where the author served as the Chief Technologist and Deputy Program Manager.

\section{References}

${ }^{1}$ Larson, C. A., Rotating Space Station Stabilization Criteria for Artificial Gravity, NASA, URL: http://ntrs.nasa.gov/archive/nasa/casi.ntrs.nasa.gov/19690029825.pdf, 1969.

${ }^{2}$ Bonestell, C., "von Braun's Space Station," In (von Braun, 1952).

${ }^{3}$ von Braun, W., "Crossing the Last Frontier," Collier's, 22 Mar. 1952, pp. 24-30, 72-74.

${ }^{4}$ Committee on Creating a Vision for Space Medicine During Travel Beyond Earth Orbit, Board on Health Sciences Policy, Safe Passage: Astronaut Care for Exploration, (J. R. Ball, and C. H. Evans Jr., Eds.) National Academic Press, URL: http://www.nap.edu/catalog/10218.html, 2001.

${ }^{5}$ International Academy of Astronautics, Study on Artificial Gravity Research to Enable Human Space Exploration, (L. Young, K. Yajima, and W. Paloski, Eds.), Cologne, DLR Institute of Aerospace Medicine, URL: https://iaaweb.org/iaa/Scientific\%20Activity/Study\%20Groups/SG\%20Commission\%202/sg22/sg22finalreportr.pdf, 2009.

${ }^{6}$ National Academies National Research Council Aeronautics and Space Engineering Board, NASA Space Technology Roadmaps and Priorities: Restoring NASA's Technological Edge and Paving the Way for a New Era in Space, Washington, D.C., The National Academies Press, 2012.

${ }^{7}$ NASA, NASA's Journey to Mars: Pioneering Next Steps in Space Exploration, Washington D.C., NASA Publications, URL: http://www.nasa.gov/sites/default/files/atoms/files/journey-to-mars-next-steps-20151008_508.pdf, 19 May 2015.

${ }^{8}$ Kelly, S. in "Human Spaceflight Ethics and Obligations: Options for Monitoring, Diagnosing, and Treating Former Astronauts," Hearing before the Subcommittee on Space, Committee on Science, Space and Technology, U.S. House of 
Representatives, URL: https://science.house.gov/sites/republicans.science.house.gov/files/documents/HHRG-114-SY16-WStateSKelly-20160615.pdf, 15 June 2016.

${ }^{9}$ Clément, G., and Bukley, A. (Eds.), Artificial Gravity, Hawthorne and New York, CA and NY: Microcosm Press and Springer, 2007.

${ }^{10}$ Matsnev, E. I., "Angular Acceleration" In C. S. Huntoon, V. V. Antipov, and A. I. Grigoriev (Eds.), Space Biology and Medicine III: Humans in Spaceflight, Vol. 3, AIAA, 1996, pp. 333-348.

${ }^{11}$ Davis-Street, J., and Paloski, W. H., Artificial Gravity: Will it Preserve Bone Health on Long-Duration Missions?, NASA, URL: http://ntrs.nasa.gov/search.jsp?R=20070028552, 2007.

${ }^{12}$ Yang, Y., Baker, M., Graf, S., Larson, J., and Caiozzo, V. J., "Hypergravity resistance exercise: the use of artificial gravity as potential countermeasure to microgravity," J Appl Physiol, 103, doi:10.1152/japplphysiol.00772.2007, 2007, pp. 1879-1887.

${ }^{13}$ Oyama, J., and Platt, W. T., "Effects of prolonged centrifugation on growth and organ development of rats," The American Journal of physiology, 1965, pp. 611-615.

${ }^{14}$ Genchi, G. G., Cialdai, F., Monici, M., Mazzolia, B., Mattoli, V., and Ciofani, G., "Hypergravity Stimulation Enhances PC12 Neuron-Like Cell Differentiation," BioMed Research International, URL: http://dx.doi.org/10.1155/2015/748121, 2015.

${ }^{15}$ Clements, F., and Shelford, V., Bio-Ecology, NY: John Wiley \& Sons, 1939.

${ }^{16}$ Whittaker, R., Communities and Ecosystems, $2^{\text {nd }}$ ed., NY: MacMillan, 1975.

${ }^{17}$ Gagarin Cosmonaut Training Center, Centrifuge. URL: Gagarin Cosmonaut Training Center, URL: http://www.gctc.su/main.php?id=131, 15 May, 2015.

${ }^{18}$ NASA ARC, "Ames Research Center 20-G Centrifuge," Space Biosciences, URL: http://www.nasa.gov/ames/research/space-biosciences/20-g-centrifuge, [cited 19 May 2015].

${ }^{19}$ Darwin, E., Zoonomia; or the Laws of Organic Life, Boston, MA, Thomas and Andrews, 1809.

${ }^{20}$ Harsch, V., "Centrifuge 'Therapy' for Psychiatric Patients in Germany in the Early 1800s," Aviation, Space, and Environmental Medicine, 77(2), Feb. 2006, pp. 157-160.

${ }^{21}$ Tsiolkovsky, K., Dreams of Earth and Sky, originally published as Grezy o Zemle i nebe i effekty vsemirnogo tjatotenija, Moscow, A N Gončarov, 1895, in A. Starchild (Ed.), The Science Fiction of Konstantin Tsiolkovsky, Honolulu, Hawaii, University Press of the Pacific, 1979, pp. 52-154.

${ }^{22}$ Andrews, J. T., Red Cosmos: K E. Tsiolkovskii, Grandfather of Soviet Rocketry, College Station, TX: Texas A\&M University Press, 2009.

${ }^{23}$ Tsiolkovsky, K. E., Investigation of World Space by Reactive Vehicles, originally published as Issledovanie mirovykh prostranstv reaktivnymi priborami, Vestnik Vozdukhoplavaniva, 1911-1912, in V. N. Sokolsky, and A. A. Blagonravov (Eds.), Selected Works of Konstantin E. Tsiolkovsky, G. Yankovsky, Trans., Second ed., Honolulu, Hawaii, University Press of the Pacific, 2004, pp. 83-139.

${ }^{24}$ Hoey, R. G., "Physical Problems, Challenges, and Pragmatic Solutions," In R. P. Hallion (Ed.), NASA's contributions to aeronautics : aerodynamics, structures, propulsion, and controls, Vol. 1, Washington, DC, NASA, URL: http://www.nasa.gov/pdf/482993main_ContributionsVolume1.pdf, 2010.

${ }^{25}$ Gerathewohl, S. J., Zero-G Devices and Weightlessness Simulators, Washington, D.C.: National Academy of Sciences National Research Council, 1961.

${ }^{26}$ NASA, AC76-0525, "Torus Sphere Space Colony Concept Illustration," URL: http://ails.arc.nasa.gov/Images/newimages/JPEGs/highres/AC76-0525.jpg, 1977.

${ }^{27}$ NASA - American Society for Engineering Education 1975 Summer Faculty Fellowship Program in Engineering Systems Design, Space Settlements: A Design Study, R. D. Johnson, and C. Holbrow, (Eds.), Washington, D.C., NASA, 1977.

${ }^{28}$ Hall, T. W., The Architecture of Artificial-Gravity Environments for Long-Duration Space Habitation, Doctoral Dissertation. Ann Arbor: University of Michigan, 1994.

${ }^{29}$ van Loon, J. J., "The Human Centrifuge.," Microgravity Science and Technology, 21(1-2), doi:10.1007/s12217-008-9062-z, Jan. 2009, pp. 203-207.

${ }^{30}$ van Loon, J. J., and Wuyts, F., "The Large Radius Human Centrifuge 'A Human Hypergravity Habitat, H3'," 60th International Astronautical Congress Space Life Sciences Symposium, Seoul, Korea, International Astronautical Federation, 2009.

${ }^{31}$ van Loon, J. J., "A large human centrifuge for exploration and exploitation research," Annales Kinesiologiae, 3(1), URL: http://ojs.zrs.upr.si/index.php/AK/article/view/67, 2012, 107-121.

${ }^{32}$ Dickinson, H., and Titley, A., Richard Trevithick: The Engineer and the Man, Cambridge: Cambridge University Press, 1934, pp. 269-278.

${ }^{33}$ Dickinson, H., and Titley, A., Richard Trevithick: The Engineer and the Man, Cambridge: Cambridge University Press, 1934, pp. 63.71.

${ }^{34}$ Amgueddfa Cymru - National Museum Wales, Richard Trevithick's steam locomotive. URL: Amgueddfa Cymru — National Museum Wales: http://www.museumwales.ac.uk/rhagor/article/trevithic_loco/, 15 Dec. 2008.

${ }^{35}$ Van Dorn, W. E., and Beemer, Patent No. 2,225,242, USA, URL: http://pimg-fpiw.uspto.gov/fdd/42/252/022/0.pdf, 1940.

${ }^{36}$ Life Magazine, "'Empire Builder' Hill's Grandson Builds a Jounceless Railroad Car," Life Magazine, 20 May 1940, pp. 41-

42.

${ }^{37}$ Schoepf, T. H., and Ritchie, D. M., Patent No. 2,088,487, USA, 1937.

${ }^{38}$ Schoepf, T. H., and Ritchie, D. M., Patent No. 2,095,677, USA, 1937. 
${ }^{39}$ Persson, R., Tilting trains: Description and analysis of the present situation Literature study, Linkoping, Swedish National Road and Transport Research Institute (VTI), URL: http://www.vti.se/en/publications/pdf/tilting-trains--description-and-analysisof-the-present-situation-a-literature-study.pdf, 2007.

${ }^{40}$ Khedkar, S. B., Kasav, S. M., Jadhav, V. S., Katkade, S. D., and Gunjal, S. U., "Tilting Train Technology," International Journal of Advanced Technology in Engineering and Science, 3(1), URL: http://www.ijates.com/images/short_pdf/1427564986_939.pdf, 2015, pp. 1561-1565.

${ }^{41}$ Bacon, K. W., and Morgan, E. O., Patent No. 3167024. USA, 1965

${ }^{42}$ Bacon, K. W., Patent No. 3889605, USA, 1975.

${ }^{43}$ Reynolds, R. R., Roller Coasters, Flumes and Flying Saucers, Northern Lights Pub., 1999.

${ }^{44}$ Ferrari World Abu Dhabi, Formula Rossa, URL: Ferrari World Abu Dhabi: http://www.ferrariworldabudhabi.com/engb/attractions/formula-rossa.aspx, 24 July 2015.

${ }^{45}$ Siemens AG., "Amtrak Cities Sprinter ACS-64 Electric Locomotive," Siemens Locomotives, URL: sitecollectiondocuments/en/rail-solutions/locomotives/customspecific-solutions/amtrak-acs64-en.pdf, [cited 25 July 2015 ].

${ }^{46}$ Hellinger, R., and Mnich, P., "Linear Motor-Powered Transportation: History, Present Status, and Future Outlook," Proceedings of the IEEE, 97, IEEE. doi:10.1109/JPROC.2009.2030249, Nov. 2009, pp. 1892-1900.

${ }^{47}$ Zehden, A., Patent No. 782312, USA, URL: http://pimg-fpiw.uspto.gov/fdd/12/823/007/0.pdf, 1905.

${ }^{48}$ Bachelet, E., Patent No. 1020942, USA, URL: http://pimg-fpiw.uspto.gov/fdd/42/209/010/0.pdf, 1912.

${ }^{49}$ Powell, J. R., and Danby, G. T., Patent No. 3470828, USA, URL: http://pimg-fpiw.uspto.gov/fdd/28/708/034/0.pdf. 1969.

${ }^{50}$ Antlauf, W., Bernardeau, F. G., and Coates, K. C., Fast Track. Civil Engineering, 74(11), Nov. 2004, pp. 35-43.

${ }^{51}$ Shanghaichina.ca, "The Shanghai Maglev Train," Shanghai China Tourist Information and Travel Guide, URL: http://shanghaichina.ca/video/maglevtrain.html, [cited 26 June 2015].

${ }^{52}$ Central Japan Railway, Central Japan Railway Company Annual Report 2014, URL: Central Japan Railway Company: http://english.jr-central.co.jp/company/ir/annualreport/_pdf/annualreport2014.pdf, 26 June, 2014.

${ }^{53}$ Guinness World Records, Fastest maglev train, Guinness World Records, URL: http://www.guinnessworldrecords.com/world-records/fastest-maglev-train/, 26 June 2015.

${ }^{54}$ Post, R. F., Patent No. 5722326, USA, URL: http://pimg-fpiw.uspto.gov/fdd/26/223/057/0.pdf, 1998

${ }^{55}$ Post, R. F., and Ryutov, D. D., "The Inductrack: A Simpler Approach to Magnetic Levitation," IEEE Transactions on Applied Superconductivity, 10(1), IEEE, doi:10.1109/77.828377, 2000, pp. 901-904.

${ }^{56}$ Powell, J. R., Maise, G., Paniagua, J., and Rather, J. D., "StarTram: A New Approach for Low-Cost Earth-to-Orbit Transport," IEEE Aerospace Conference Procs, 5, Big Sky, MT, IEEE, doi:10.1109/AERO.2001.931219, 2001, , pp. $2569-2591$.

${ }^{57}$ Powell, J., Maise, G., and Pellegrino, C., StarTram: The New Race to Space, CreateSpace Independent Publishing, 2013.

${ }^{58}$ U.S. Federal Government, Code of Federal Regulations Title 46, Vols. 4, Part 115-114, Washington, D.C., U.S. Government Printing Office, URL: http:/www.gpo.gov/fdsys/pkg/CFR-2011-title46-vol4/pdf/CFR-2011-title46-vol4part115.pdf, 2011.

${ }^{59}$ Royal Caribbean International, Adventure of the Seas, URL: Royal Caribbean International: http://www.royalcaribbean.com/findacruise/ships/class/ship/home.do?shipCode=AD, [cited 4 June 2015].

${ }^{60}$ Kable Intelligence Limited, Adventure of the Seas, Voyager-Class Cruise Ship, Ship-Technology.com, URL: http://www.ship-technology.com/projects/adventure/, [cited 10 May 2015].

${ }^{61}$ Cybercruises.com, Mariner of the Seas, the fifth cruise ship in the Voyager-class for Royal Caribbean Cruises was delivered by Kvaerner Masa-Yards, URL: Cybercruises.com: http://www.cybercruises.com/masethficrshnov03.htm, 4 Nov. 2003.

${ }^{62}$ Trauthwein, G., "Navigator of the Seas -- A New Course for Profitability." Maritime Reporter and Engineering News, URL: http://magazines.marinelink.com/magazines/MaritimeReporter/200302/pdf/, Feb. 2003, pp. 24-27.

${ }^{63}$ MarineLink.com, World's Largest Cruise Ship Launched, URL: http://www.marinelink.com/news/article/world-s-largestcruise-ship-launched/307105.aspx, 19 Aug. 2005.

${ }^{64}$ Maidl, B., Schmid, L., Ritz, W., and Herrenknecht, M., Hardrock Tunnel Boring Machines, Berlin, Ernst and Sohn, 2008

${ }^{65}$ Zhao, J., "A General Overview on Tunnel Boring Machines," Society for Rock Mechanics \& Engineering Geology Seminar, Singapore, Nanyang Technological University, URL: http://www.ncus.ntu.edu.sg/Events/Documents/SRMEG\%20Seminar\%20Tunnel\%20Boring\%20Machines.pdf, 2012.

${ }^{66}$ Kirk, J., "Fortescue opens the world's heaviest haul railway," Railway Gazette, URL: http://www.railwaygazette.com/news/single-view/view/fortescue-opens-the-worlds-heaviest-haul-railway.html, 14 July, 2008.

${ }^{67}$ Sanyal, A. K., Shen, J., McClamroch, N. H., and Bloch, A. M., "Stability and Stabilization of Relative Equilibria of Dumbbell Bodies in Central Gravity," Journal of Guidance, Control, and Dynamics, 28(5), doi:10.2514/1.10546, Sept.-Oct. 2005, pp. 833-842.

${ }^{68}$ McConaghy, T. T., Longuski, J. M., and Byrnes, D. V., "Analysis of a Broad Class of Earth-Mars Cycler Trajectories," AIAA Astrodynamics Specialist Conference, Monterey, AIAA/AAS, 2002, pp. 1-10. 